\title{
The Galactic Magneto-ionic Medium Survey: Moments of the Faraday Spectra
}

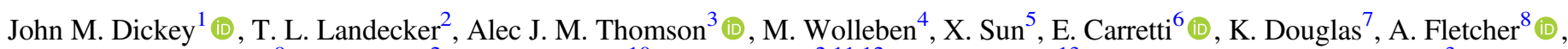 \\ B. M. Gaensler ${ }^{9}$ (D) A. Gray ${ }^{2}$, M. Haverkorn ${ }^{10}$ (D), A. S. Hill ${ }^{2,11,12}$ (D), S. A. Mao ${ }^{13}$, and N. M. McClure-Griffiths ${ }^{3}$ (D) \\ ${ }^{1}$ School of Natural Sciences, Private Bag 37, University of Tasmania, Hobart, TAS, 7001, Australia; john.dickey@utas.edu.au \\ ${ }^{2}$ National Research Council Canada, Dominion Radio Astrophysical Observatory, P.O. Box 248, Penticton, British Columbia, V2A 6J9, Canada \\ ${ }^{3}$ Research School of Astronomy and Astrophysics, Australian National University, Canberra, ACT 2611, Australia \\ ${ }^{4}$ Skaha Remote Sensing Ltd., 3165 Juniper Drive, Naramata, BC VOH 1NO, Canada \\ ${ }^{5}$ Department of Astronomy, Yunnan University, and Key Laboratory of Astroparticle Physics of Yunnan Province, Kunming, 650091, People's Republic of China \\ ${ }^{6}$ INAF-Instituto de Radioastronomia, Via P. Gobetti 101, I-40129, Bologna, Italy \\ ${ }_{8}^{7}$ Physics and Astronomy Department, Okanagan College, 1000 KLO Road, Kelowna, BC V1Y 4X8, Canada \\ ${ }^{8}$ School of Mathematics, Statistics and Physics, Newcastle University, Newcastle-upon-Tyne, NE13 7RU, UK \\ ${ }^{9}$ Dunlap Institute for Astronomy and Astrophysics, University of Toronto, Toronto, ON M5S 3H4, Canada \\ ${ }^{10}$ Department of Astrophysics/IMAPP, Radboud University, P.O. Box 9010, NL-6500 GL Nijmegen, The Netherlands \\ ${ }^{11}$ Department of Physics and Astronomy, University of British Columbia, Vancouver, BC V6T 1Z1, Canada \\ ${ }^{12}$ Space Science Institute, Boulder, CO 80301, USA \\ ${ }^{13}$ Max Planck Institute for Radio Astronomy, Auf dem Hügel 69, D-53121 Bonn, Germany \\ Received 2018 October 11; revised 2018 November 24; accepted 2018 December 11; published 2019 January 25
}

\begin{abstract}
Faraday rotation occurs along every line of sight in the Galaxy; rotation measure (RM) synthesis allows a 3D representation of the interstellar magnetic field. This study uses data from the Global Magneto-Ionic Medium Survey, a combination of single-antenna spectro-polarimetric studies, including northern sky data from the Dominion Radio Astrophysical Observatory (DRAO) $26 \mathrm{~m}$ telescope $(1270-1750 \mathrm{MHz}$ ) and southern sky data from the Parkes $64 \mathrm{~m}$ telescope $(300-480 \mathrm{MHz})$. From the synthesized Faraday spectral cubes we compute the zeroth, first, and second moments to find the total polarized emission, mean RM, and RM width of the polarized emission. From DRAO first moments we find a weak vertical field directed from Galactic North to South, but Parkes data reveal fields directed toward the Sun at high latitudes in both hemispheres: the two surveys clearly sample different volumes. DRAO second moments show feature widths in Faraday spectra increasing with decreasing positive latitudes, implying that longer lines of sight encounter more Faraday rotating medium, but this is not seen at negative latitudes. Parkes data show the opposite: at positive latitudes the second moment decreases with decreasing latitude, but not at negative latitudes. Comparing first moments with RMs of pulsars and extragalactic sources and a study of depolarization together confirm that the DRAO survey samples to larger distances than the Parkes data. Emission regions in the DRAO survey are typically 700-1000 pc away, slightly beyond the scale height of the magneto-ionic medium; emission detected in the Parkes survey is entirely within the magneto-ionic disk, less than $500 \mathrm{pc}$ away.
\end{abstract}

Key words: cosmic rays - ISM: magnetic fields - local interstellar matter - techniques: polarimetric

\section{Introduction}

\subsection{Galactic Diffuse Polarized Emission}

The magnetic field of the Milky Way can be traced qualitatively and measured quantitatively through various observations, many of them involving polarization (Ferrière 2015; Mao et al. 2015a; Han 2017; Planck Collaboration et al. 2018). Starlight polarization that shows large-scale patterns, and generally increases with the distance of the star, was the first evidence for a coherent magnetic field on a large scale in the Galactic interstellar medium (Hall 1949; Hiltner 1949; Mathewson \& Ford 1970), and it remains a valuable tracer of the magnetic field configuration on various scales (Heiles 2000). The same large-scale alignment of spinning, aspherical dust grains that causes the starlight polarization causes polarized far-infrared emission (Houde et al. 2011). In the nearinfrared, starlight polarization allows the field configuration to be traced further into dark interstellar clouds (Jones 2003; Clemens et al. 2012). Quantitative measurement of the line-of-sight component of the magnetic field is possible with Zeeman splitting observations of various spectral lines; the $21 \mathrm{~cm}$ line of atomic hydrogen is the most widespread, and it provides opportunities to measure the splitting either in absorption or in emission (e.g., Crutcher et al. 2010). One of the most widespread tracers of the
Galactic magnetic field is radio synchrotron emission, which is linearly polarized owing to the motion of the relativistic electrons around the magnetic field lines. The fairly strong and consistent linear polarization of the Galactic diffuse emission at radio frequencies was one of the first and most convincing arguments in favor of the synchrotron emission process (Alfvén \& Herlofsen 1950), reviewed by Ginzburg \& Syrovatskii (1965).

At radio frequencies, linearly polarized emission propagating through an ionized medium with a magnetic field that has a component along the line of sight will show a rotation of the plane of polarization due to Faraday rotation (e.g., Harwit 1973, chap. 6; Jokipii \& Lerche 1969). The position angle, $\chi$, of the polarization is defined in terms of the Stokes parameters $Q$ and $U$, as

$$
\chi=\frac{1}{2} \arctan \left(\frac{U}{Q}\right)
$$

where the signs of both $U$ and $Q$ are used to determine $\chi$ over the full $\pm \pi$ phase range. The position angle changes with wavelength, $\lambda$; for any value of $\lambda^{2}$ we can measure the derivative,

$$
\mathrm{RM}=\frac{d \chi}{d\left(\lambda^{2}\right)}
$$


in units of radians per meter squared. This empirical definition allows many different values of rotation measure $(\mathrm{RM})$ to be present in a single complex spectrum of $Q+i U$ as a function of $\lambda^{2}$.

Many compact polarized sources show a single value of RM, which can be interpreted as the effect of magnetized plasma along the line of sight from the source at distance $d$ to the observer (at distance zero):

$$
\mathrm{RM}=0.81 \int_{d}^{0} n_{e} \boldsymbol{B} \cdot d \boldsymbol{s} .
$$

If $n_{e}$, the electron density, is in units of $\mathrm{cm}^{-3}, B$, the magnetic field, is in $\mu \mathrm{G}$, and $d$ is in pc, then RM is given by Equation (1) in $\mathrm{rad} \mathrm{m}^{-2}$. The convention that $d s$ points along the line of sight from the source to the observer in Equation (1) sets the convention that $\mathrm{RM}$ is positive for the $B$ field pointing toward the observer.

Over the past decade, surveys of RMs of larger and larger samples of extragalactic continuum sources have been made, some concentrating on low Galactic latitudes (Han 2017, Figure 8) and others covering all the sky available to the telescope (Stil et al. 2011). These have been combined by Oppermann et al. $(2012,2015)$ into a grid of the best estimates for the Galactic contribution to the RM in each cell on the sky. Since the individual sources have intrinsic RMs as well as the Galactic $\mathrm{RM}$, the precision of the estimate of the Galactic foreground depends on the density of point sources. Future surveys such as POSSUM (Gaensler 2009) will greatly improve the precision of maps like those of Oppermann et al. Surveys of the RM of extragalactic radio sources show large-scale patterns at high (Taylor et al. 2009; Mao et al. 2010, 2012; Mao 2018) and low latitudes (Ordog et al. 2017), somewhat similar to those seen in the starlight polarization. RM surveys of pulsars are particularly valuable, because the RM divided by the dispersion measure, $\mathrm{DM}=\int_{0}^{d} n_{e} d s$, provides a measure of the $\overline{B_{\|}}=\mathrm{RM} / \mathrm{DM}$ averaged along the line of sight (Yao et al. 2017; Han et al. 2018a).

\subsection{The Faraday Depth ( $\phi)$ Axis}

In contrast to the RM, the Faraday depth, $\phi$, is an independent variable with units of $\mathrm{rad} \mathrm{m}^{-2}$ over which we compute the distribution of linearly polarized brightness as the Faraday spectrum, the polarized intensity $F$, as a function of $\phi$,

$$
F(\phi)=\frac{1}{\pi} \int_{-\infty}^{+\infty} P\left(\lambda^{2}\right) e^{-2 i \phi \lambda^{2}} d\left(\lambda^{2}\right)
$$

(Burn 1966, Equation (11)). A broadband polarization survey of $Q$ and $U$ over a wide range of $\lambda$ can be transformed into a Faraday depth cube. This is analogous to a spectral line cube for which the axes are two sky coordinates and Doppler velocity (measured as frequency or wavelength). For linear polarization surveys, the third axis is not velocity but Faraday depth, $\phi . F(\phi)$ is the Fourier conjugate function to $P\left(\lambda^{2}\right)$; it is also complex, with real part Stokes $Q(\phi)$ and imaginary part Stokes $U(\phi)$. The Faraday spectrum may be represented as polarized brightness temperature:

$$
T(\phi)=|F|=\sqrt{Q(\phi)^{2}+U(\phi)^{2}},
$$

where $Q, U, F, P$, and $T$ all have units of $\mathrm{K}$ since the diffuse emission is calibrated as brightness temperature using the
Rayleigh-Jeans approximation. The symbol $T_{p}$ is often used for the linearly polarized brightness temperature, to distinguish it from the unpolarized emission; in this paper we do not discuss the Stokes $I$ or $V$ parameters at all, so we can abbreviate $T_{p}$ by simply $T$. As functions of $\phi$, or Faraday spectra, the true distributions of these quantities are distorted by the resolving function or RM spread function (RMSF) that is determined by the limited range of wavelength squared in the observations. This distortion can be partially corrected by deconvolution with the RM-CLEAN algorithm (Heald 2009), which changes the resolving function from a messy dirty beam to a smoother clean beam that is chosen to be a Gaussian. The polarized brightness temperature in the cleaned spectrum then has units of $\mathrm{K}$ beam $^{-1}$, where the beam is the clean RMSF used in the deconvolution process. For brevity we will use simply $\mathrm{K}$ units for $T(\phi)$.

It is only since the mid-2000s that the necessary parameters of a survey of diffuse polarization have been understood. This is because the requirements of bandwidth and resolution imposed by the Fourier relationship between $F(\phi)$ and $P\left(\lambda^{2}\right)$, derived originally by Burn (1966), were not widely appreciated until the seminal paper by Brentjens \& de Bruyn (2005). An ambitious international collaboration to use large, single-dish radio telescopes with broadband spectro-polarimeters to determine the structure of the Galactic magneto-ionic medium was begun in 2008, called GMIMS (the Galactic MagnetoIonic Medium Survey; Wolleben et al. 2009). GMIMS uses the variation of the strength of the Stokes $Q$ and $U$ components with $\lambda^{2}$ through the Fourier transform to determine the distribution of the polarized emission as a continuous function of $\phi$ (de Bruyn \& Brentjens 2005).

Two of the GMIMS surveys have been completed, and the data are fully reduced and calibrated: the Dominion Radio Astrophysical Observatory (DRAO) survey of the northern sky $\left(87^{\circ}>\delta>-30^{\circ}\right)$ at frequencies $1270-1750 \mathrm{MHz}$ and the Parkes survey of the southern sky $\left(-90^{\circ}<\delta<+20^{\circ}\right)$ at frequencies $300-480 \mathrm{MHz}$ (Wolleben et al. 2018). The corresponding wavelength and RM coverage are summarized in Table 1 . The numbers in Table 1 are computed using the full bandwidth used to construct the Faraday cube. In some directions some spectral channels were flagged as a result of interference. This flagging causes variation in the parameters in Table 1 from place to place in the two Faraday cubes.

\subsection{The RM Spread Function}

If the spectrometer provides a bandwidth and channel separation translated to wavelength squared that has some sensitivity function, $W\left(\lambda^{2}\right)$ in the notation of Brentjens \& de Bruyn (2005), then the resolving function in the RM dimension is the Fourier transform of $W$. This is the RMSF, $R(\phi)$. For a simple $W\left(\lambda^{2}\right)$ that is a top-hat (boxcar) function centered on $\lambda_{c}^{2}$ with width $\Delta \lambda^{2}=\lambda_{2}^{2}-\lambda_{1}^{2}$, the corresponding $R$ is a sinc function with a phase wind:

$$
R(\phi)=e^{i\left(\phi \lambda_{c}^{2}\right)} \frac{\sin \left(\phi \Delta \lambda^{2}\right)}{\phi \Delta \lambda^{2}}
$$

(illustrated in Appendix B). Note that $\Delta \lambda^{2}$ indicates $\Delta\left(\lambda^{2}\right)$ rather than $(\Delta \lambda)^{2}$. The width of a $\operatorname{sinc}(\theta)$ function measured between half-power points is $\delta \theta=3.79$, so the resolution in $\phi$ of the survey is roughly the width of the main lobe of the 
Table 1

Survey Details

\begin{tabular}{|c|c|c|c|c|}
\hline \multirow{2}{*}{ Survey } & \multicolumn{2}{|c|}{ Parkes } & \multicolumn{2}{|c|}{ DRAO } \\
\hline & $\min$ & $\max$ & $\min$ & $\max$ \\
\hline Decl. range & $-90^{\circ}$ & $+20^{\circ}$ & $-30^{\circ}$ & $+87^{\circ}$ \\
\hline Angular resolution & $83 ! 6$ & $79 ! 4$ & $40^{\prime}$ & $30 ! 5$ \\
\hline Frequency range & $300.25 \mathrm{MHz}$ & $479.75 \mathrm{MHz}$ & $1270 \mathrm{MHz}$ & $1750 \mathrm{MHz}$ \\
\hline$\lambda^{2}$ range & $0.391 \mathrm{~m}^{2}$ & $1.0 \mathrm{~m}^{2}$ & $0.029 \mathrm{~m}^{2}$ & $0.056 \mathrm{~m}^{2}$ \\
\hline$\Delta \lambda^{2}$ & $0.608 \mathrm{~m}^{2}$ & $\ldots$ & $0.026 \mathrm{~m}^{2}$ & \\
\hline$\delta \lambda^{2}$ & $3.32 \cdot 10^{-3} \mathrm{~m}^{2}$ & $\ldots$ & $6.2 \cdot 10^{-5} \mathrm{~m}^{2}$ & $\ldots$ \\
\hline RM resolution $\delta \phi$ & $6.2 \mathrm{rad} \mathrm{m}^{-2}$ & $\cdots$ & $1.4 \cdot 10^{2} \mathrm{rad} \mathrm{m}^{-2}$ & $\ldots$ \\
\hline RM range $\phi_{\max }$ & $1.3 \cdot 10^{3} \mathrm{rad} \mathrm{m}^{-2}$ & $\cdots$ & $3.1 \cdot 10^{4} \mathrm{rad} \mathrm{m}^{-2}$ & $\ldots$ \\
\hline RM feature width $\phi_{\text {max-scale }}$ & $8.0 \mathrm{rad} \mathrm{m}^{-2}$ & $\ldots$ & $1.1 \cdot 10^{2} \mathrm{rad} \mathrm{m}^{-2}$ & $\ldots$ \\
\hline Cleaned $\phi$ spectral range & $-100 \mathrm{rad} \mathrm{m}^{-2}$ & $+100 \mathrm{rad} \mathrm{m}^{-2}$ & $-400 \mathrm{rad} \mathrm{m}^{-2}$ & $+400 \mathrm{rad} \mathrm{m}^{-2}$ \\
\hline Faraday spectrum channel width & $0.5 \mathrm{rad} \mathrm{m}^{-2}$ & $\cdots$ & $5 \mathrm{rad} \mathrm{m}^{-2}$ & $\ldots$ \\
\hline
\end{tabular}

function $R(\phi)$, which for the simple form of Equation (3) has full width to half-maximum:

$$
\delta \phi=\frac{3.79}{\Delta \lambda^{2}}
$$

Similarly, the maximum RM that can be detected is one that would give a drop of a factor of one-half over a single step $\delta \lambda^{2}$ in the spectrum:

$$
\phi_{\max }=\frac{1.9}{\delta \lambda^{2}}
$$

As Schnitzeler \& Lee (2015) explain, the upper limit $\phi$ is somewhat lower than this depending on the computational approach taken to compute the Faraday spectrum, i.e., the discrete form of Equation (2). Their Equation (14) gives slightly lower values of $\phi_{\max }$ of $9.84 \times 10^{2} \mathrm{rad} \mathrm{m}^{-2}$ and $2.92 \times 10^{4} \mathrm{rad} \mathrm{m}^{-2}$ for the Parkes and DRAO surveys, respectively.

Since the $\phi$-axis of a Faraday spectrum is the Fourier conjugate of the $\lambda^{2}$ spectrum derived from the spectrometer output, the relationship between the spectrometer sensitivity, in $\lambda^{2}$ space, and the RMSF in $\phi$ space is similar to the relationship in aperture synthesis between the extent or coverage of observed baselines in $u, v$ space and the beam, or resolving function, in two dimensions on the plane of the sky. If there is a broad emission feature in Faraday space, the absence of the "zero-spacing" or infinite frequency measurement means that the observed Faraday spectrum is high-pass filtered, so that the edges of the broad feature are enhanced, but the rest is attenuated nearly to zero. The broadest feature that is not attenuated in this way has width $\phi_{\text {max-scale, given by }}$

$$
\phi_{\max -\text { scale }} \simeq \frac{\pi}{\lambda_{1}^{2}}
$$

An additional complication is the spectral index of the synchrotron emission, which generally has a power law with polarized brightness temperature $T(\nu) \propto \nu^{-\beta}$. This can lead to enhanced sidelobes in the uncleaned Faraday spectrum (Schnitzeler 2018, Figure 1).

The aim of the GMIMS surveys is to make $\delta \phi$ less than $\phi_{\text {max-scale }}$ for the first time at frequencies above $250 \mathrm{MHz}$ in the Milky Way. The weakness of polarization surveys taken with narrowband receivers is that the RMSF function is broader than the maximum detectable scale in $\phi$. This happens whenever the bandwidth, $\Delta \lambda^{2}$, is less than the minimum wavelength squared, $\lambda_{1}^{2}$. The result is that even a relatively simple $\phi$ spectrum is converted into a messy function; see examples in Appendix B and other examples in Appendix 2 of Brentjens \& de Bruyn (2005). As Table 1 shows, the Parkes survey has $\frac{\Delta \lambda^{2}}{\lambda_{1}^{2}}$ of about 1.6, which is quite safe. For the DRAO survey the value is $\sim 0.9$, so the RMSF is marginally affected by missing largescale Fourier components. Features in the Faraday spectra that are much wider than $\phi_{\text {max-scale }}$ will still be hollowed out, i.e., edge-filtered by the RMSF (see Appendix B). Surveys with the LOFAR and MWA telescopes at low frequencies $(\nu<250$ $\mathrm{MHz}$ ) have achieved $\delta \phi<\phi_{\max \text {-scale }}$ (Iacobelli et al. 2013; Jelić et al. 2014, 2015; Lenc et al. 2016; van Eck et al. 2017), providing resolved features in the Faraday spectrum similar to those in the Parkes survey discussed below.

In this paper we study the Faraday cubes of the two GMIMS surveys by computing the moments of the emission spectra and comparing them with other RM tracers. This is the first application of spectral moment techniques to the study of the diffuse polarized emission from the Galaxy. Note that a different set of parameters, also called Faraday moments, is proposed by Farnes et al. (2018) as statistical parameters to develop an optimal detection strategy for finding sources of polarized emission in the presence of radiometer noise. These are computed directly from $Q(\lambda)$ and $U(\lambda)$ for efficiency in searching large survey data sets.

Representative spectra from the Faraday cubes of the two surveys are presented in Section 2, the method of calculating the moments is discussed, and the zeroth, first, and second moments are shown for the full areas of the two surveys. These are 2D representations of the survey data that can be easily compared with other RM data in Section 3. In particular, comparison with RMs of nearby pulsars with known distances provides a distance estimate for the polarized emission in the DRAO survey, but not for the Parkes data, as discussed in Section 4 . The very different skies seen in the two surveys can be explained as the result of the polarization horizon, i.e., the limit to the distance from which polarized emission can reach us, determined by depolarization processes (Uyaniker et al. 2003), with the result that they sample quite different volumes, as discussed in Section 5. 


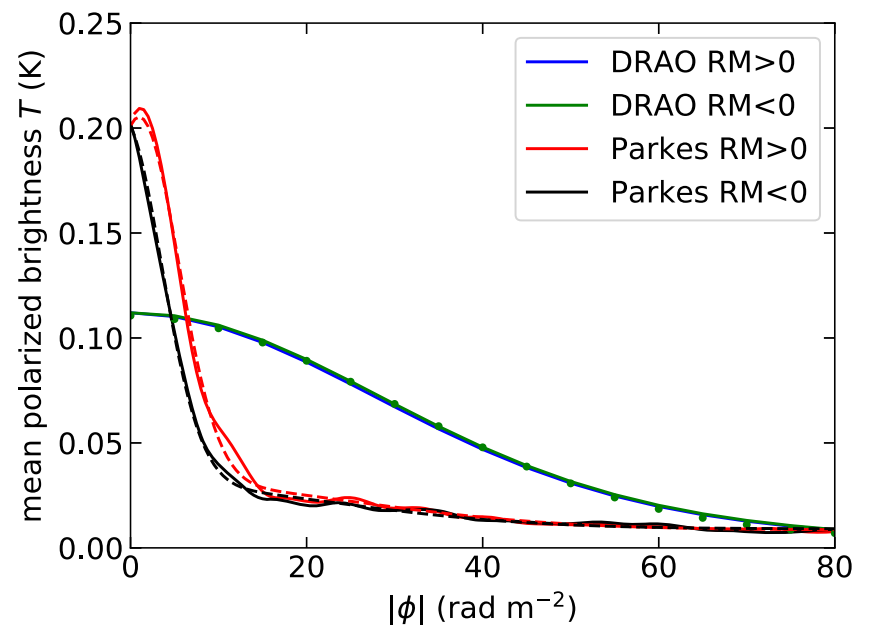

Figure 1. Average linearly polarized brightness, $T(\phi)$, over the area of each survey, computed separately for each plane of the Faraday cube. The fitted Gaussians are indicated by the green dots (DRAO) and the red and black dashed lines (Parkes) using the parameters in Table 2. The DRAO fit is a single Gaussian, whereas the Parkes fit is the sum of two Gaussians, each of the form in Equation (4). The error in $T(\phi)$ is dominated by fluctuations introduced by the limited wavelength coverage, and at low latitudes by leakage of Stokes $I$ into Stokes $Q$ and $U$. In this figure, the residuals about the best-fit Gaussian in the Parkes data are $\sim 4 \times 10^{-3} \mathrm{~K}$, and in the DRAO data they are $\sim 1 \times 10^{-3} \mathrm{~K}$.

\section{The Survey Data}

\subsection{All Sky Averages}

The Parkes and DRAO surveys are very complementary in several ways. The DRAO telescopes in British Columbia can observe the entire northern sky, and in the south down to $\delta \simeq-30^{\circ}$, and the Parkes telescope in New South Wales can observe the entire southern sky, and in the north as high as $\delta \simeq+20^{\circ}$; thus, there is an overlap band of width about $50^{\circ}$. Since the ranges of $\lambda^{2}$ are so different, the RMSFs of the two surveys are very different also (Table 1). Most importantly, the synchrotron emission has spectral index $\beta \sim-2.75$, so the much lower frequencies of the Parkes survey see brighter emission. That emission is spread over a much narrower range of $\phi$ than for the higher frequencies of the DRAO survey. This is shown in Figure 1, which plots the mean brightness temperatures of the polarized intensity of the two surveys as functions of $\phi$, averaged over the entire survey areas.

The $x$-axis of Figure 1 is $|\phi|$, to make the symmetry between the positive and negative values of $\phi$ clear, although the fitting was done for the full range. The $y$-axis plots the mean of $T(\phi)$ over the full area of each survey. The DRAO survey does not resolve the structure of the emission in Faraday depth when averaged over the full area, but when individual Faraday spectra are measured, or Faraday cubes for small regions, then structure appears, as shown in Section 2.2 below. The DRAO survey average profile is very well fit by a Gaussian as

$$
T(\phi)=T_{o} e^{\left(-\frac{\left(\phi-\phi_{o}\right)^{2}}{2 \sigma_{\phi}^{2}}\right)} .
$$

Least-squares fitted values of the Gaussian parameters are given in Table 2. The width of the DRAO Faraday spectrum is artificially made smaller than the nominal resolution of the survey (Table 1) because in the Faraday cleaning step of the data reduction the "clean beam" or restoring function was set as
Table 2

Survey Mean $T(\phi)$ Gaussian Fits

\begin{tabular}{lcrcc}
\hline \hline Survey & $\phi_{o}$ & $\sigma_{\phi}$ & $T_{o}$ & Baseline \\
\hline DRAO & $-0.3 \mathrm{rad} \mathrm{m}^{-2}$ & $30.3 \mathrm{rad} \mathrm{m}^{-2}$ & $0.11 \mathrm{~K}$ & $0.004 \mathrm{~K}$ \\
Parkes 1 & $+1.0 \mathrm{rad} \mathrm{m}^{-2}$ & $4.5 \mathrm{rad} \mathrm{m}^{-2}$ & $0.17 \mathrm{~K}$ & $0.008 \mathrm{~K}$ \\
Parkes 2 & $+1.7 \mathrm{rad} \mathrm{m}^{-2}$ & $23.5 \mathrm{rad} \mathrm{m}^{-2}$ & $0.02 \mathrm{~K}$ & $\cdots$ \\
\hline
\end{tabular}

a Gaussian of width $60 \mathrm{rad} \mathrm{m}^{-2}$. The RMSF of the Parkes survey is much narrower, and it allows resolution of two Gaussian components in the survey average Faraday spectrum, one with half-width $\sigma_{\phi}=4.5 \mathrm{rad} \mathrm{m}^{-2}$ and the second fainter but much broader with $\sigma_{\phi}=23.5 \mathrm{rad} \mathrm{m}^{-2}$ (Table 2). The polarized brightness measured in these two surveys has not been debiased to reduce the contribution of noise to $T(\phi)$; there is a nonzero baseline that is fitted along with the Gaussian parameters (fifth column, Table 2).

\subsection{Sample Faraday Spectra}

Figures 2-4 show six example RM spectra. The first two (Figure 2) are in the first quadrant, at longitudes $\ell \sim 11^{\circ}$ and $31^{\circ}$; the rest are in the outer galaxy. All are at intermediate latitudes (here meaning roughly $15^{\circ}<|b|<40^{\circ}$ ), the first four at $|b| \sim 33^{\circ}-35^{\circ}$, the last two at $|b| \sim 26^{\circ}$ and $\sim 20^{\circ}$. These directions are all in the overlap region covered by both the Parkes and DRAO surveys. They are in the directions of pulsars with distances less than $1 \mathrm{kpc}$, and with measured values of RM as discussed below in Section 3.1. For comparison, Faraday spectra at lower latitudes $\left(b=10^{\circ} .6\right)$ have been studied in detail by van Eck et al. (2017, Figure 6) with better spatial resolution and excellent RMSF cleaning.

The effect of smoothing in $\phi$ in the DRAO spectra is clear in Figures 2-4. The Parkes spectra have much higher resolution in $\phi$. But the two spectra are not consistent with each other even after accounting for the different resolutions. This is because of the very different wavelength ranges; the path lengths sampled by the two spectra are therefore very different, with the shorter wavelengths sensitive to much greater distances owing to depolarization, discussed in Section 5 below. In some cases, such as those shown in Figures 2 and 3, the peak of the DRAO feature corresponds well with the pulsar RM. This is not always the case, as discussed in Section 3.1 below.

Some of the weaker features in the Parkes spectra are very likely real, but determining the dynamic range of the Faraday spectrum, i.e., the ratio of the brightest spurious feature to the peak of the brightest feature, will require more careful analysis of both Faraday cubes (A. Ordog et al. 2018, in preparation; A. J. M. Thomson et al. 2018, in preparation).

Figures 2-4 show only the middle channels $\left(-60 \mathrm{rad} \mathrm{m}^{-2}<\phi<+60 \mathrm{rad} \mathrm{m}^{-2}\right)$ of the Faraday cube. The Gaussian features in the DRAO spectra extend to at least \pm 100 $\mathrm{rad} \mathrm{m}^{-2}$, and the Parkes spectra show some features outside this RM range as well. The full $\phi$ ranges of the cleaned Faraday cubes are \pm 100 and $\pm 400 \mathrm{rad} \mathrm{m}^{-2}$ for the Parkes and DRAO surveys, respectively (Table 1).

\subsection{Faraday Moments}

The distribution of polarized brightness on the sky shows interesting structures on a range of angular scales. Distinct structures in the GMIMS surveys have been studied individually 

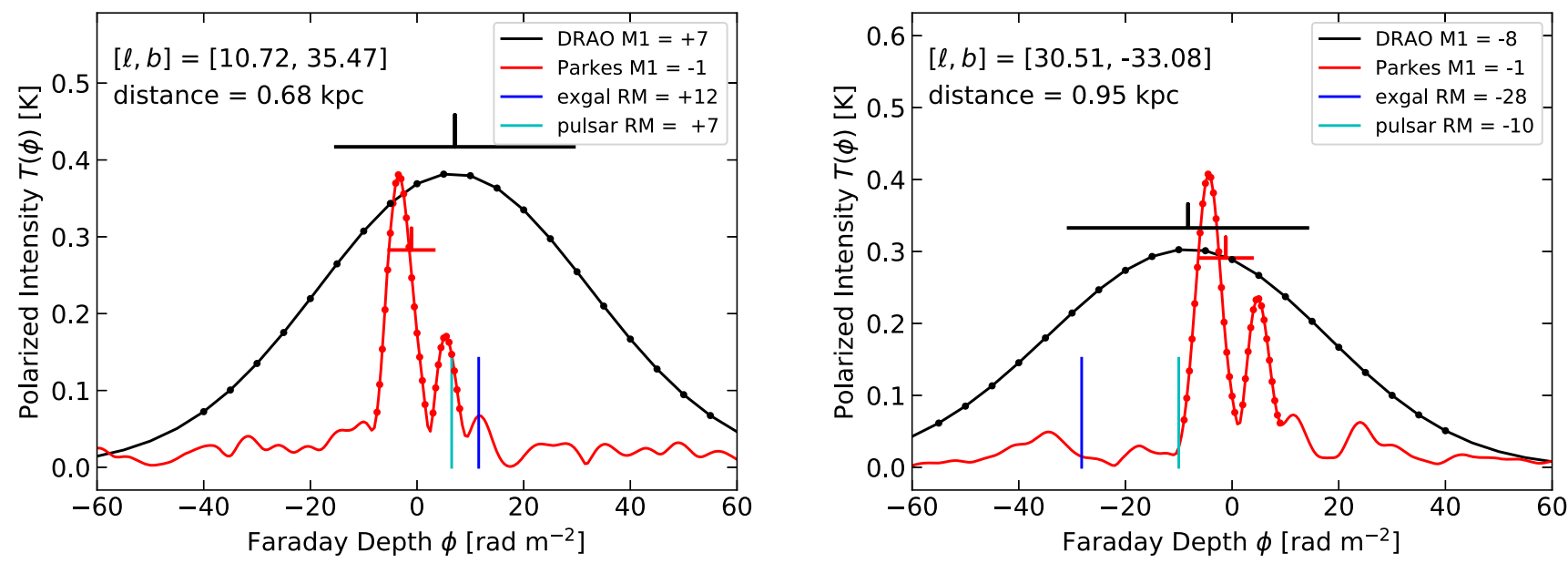

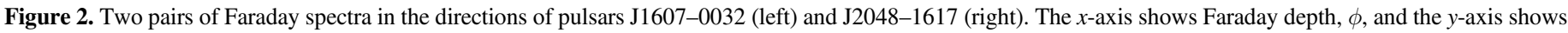

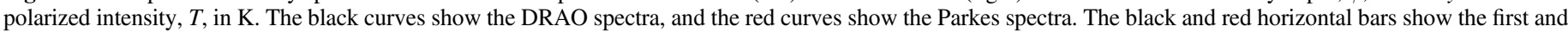

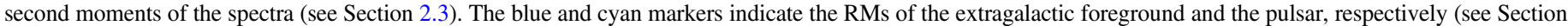

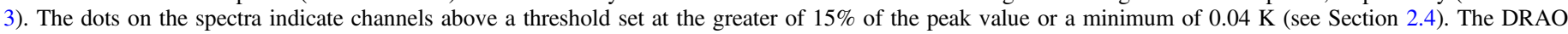
spectra generally show only one spectral feature, while the Parkes spectra often show two or more features.

(Wolleben et al. 2010; Sun et al. 2015; Hill et al. 2017; Thomson et al. 2018a), but the purpose of this paper is to study the properties of the entire sky in polarized emission, rather than individual objects. To study the properties of the Faraday cube over a large area, the spectral moments are useful tools. These are analogous to moments in velocity space for a spectral line cube. The zero moment, $M_{0}$, is defined as

$$
M_{0} \equiv \sum_{i=1}^{n} T_{i} d \phi,
$$

with units $\mathrm{K} \mathrm{rad} \mathrm{m}^{-2}$, where $d \phi$ is the width of each of the $n$ channels of the Faraday spectrum contributing to the sum. The first moment, $M_{1}$, is defined as

$$
M_{1} \equiv \frac{\sum_{i=1}^{n} T_{i} \cdot \phi_{i}}{\sum_{i=1}^{n} T_{i}}
$$

with units $\operatorname{rad~m}^{-2}$. The second moment, $M_{2}$, is defined as

$$
M_{2} \equiv \frac{\sum_{i=1}^{n} T_{i} \cdot\left(\phi_{i}-M_{1}\right)^{2}}{\sum_{i=1}^{n} T_{i}},
$$

with units $\left(\mathrm{rad} \mathrm{m}^{-2}\right)^{2}$. The sums are taken over the channels of the $\phi$ spectrum, or selected ranges of channels where the signal is well above the noise, and $T_{i}$ is the polarized intensity, $T\left(\phi_{i}\right)$, in brightness temperature units. For a continuous distribution, $T(\phi)$, the moments are integrals, $M_{0}=\int_{-\infty}^{\infty} T(\phi) d \phi$, $M_{1}=\frac{\int_{-\infty}^{\infty} T(\phi) \cdot \phi d \phi}{M_{0}}$, and $M_{2}=\frac{\int_{-\infty}^{\infty} T(\phi) \cdot\left(\phi-M_{1}\right)^{2} d \phi}{M_{0}}$. For a single Gaussian spectral feature with no noise, the moments correspond to $M_{0}=\sqrt{2 \pi} T_{o} \sigma_{\phi}, M_{1}=\phi_{o}$, and $M_{2}=\sigma_{\phi}^{2}$. To simplify comparison between the moments, we compute the square root of the second moment, $m_{2}=\sqrt{M_{2}}$; all plots involving second moments in this paper use $m_{2}$ for the second moment, with dimension $\operatorname{rad~m}^{-2}$. Note that $M_{2}$ is the second central moment, because it is taken about the mean, $M_{1}$. The effect of taking the moments is to reduce the Faraday cube to a series of images, having just the two angular dimensions of the survey, but with the images representing the distribution of brightness over the third dimension, $\phi$. Simpler alternatives to the spectral moments are discussed in Appendix A.

For an intuitive understanding, the zero moment is the total polarized brightness integrated over the full range of $\phi$, the first moment is the intensity weighted mean of $\phi$, and the square root of the second moment, $m_{2}$, is the half-width of the brightness distribution along the $\phi$-axis. Neither the peak $T(\phi)$ nor the value of $\phi$ at the peak is measured by the moments, although $T_{o}$ can be estimated assuming a Gaussian or other functional form for the line shape. The red and black bars in Figures 2-4 are placed at the height of an equivalent Gaussian profile with the same $M_{0}$ and $m_{2}$ values, which is $T_{\text {peak }}=\frac{M_{0}}{\sqrt{2 \pi} m_{2}}$.

\subsection{Thresholding}

Because of the weighting by $\phi_{i}$ and $\left(\phi_{i}-M_{1}\right)^{2}$ in Equations (6) and (7), the first and second moments are strongly affected by noise or spurious features in the spectra at high positive and negative values of $\phi$. Since $T_{i}$ is positive definite, this is an even worse problem for computing the moments of Faraday spectra than it is for more familiar velocity spectra, which are usually dominated by Gaussian noise. In most directions in both of the surveys considered here, the noise is primarily from residuals left by the Faraday deconvolution process. To mitigate the effect of spurious emission at high positive and negative values of $\phi$, we use a threshold to restrict the range of channels contributing to the sums in Equations (5)-(7).

For each pixel in the cube, the thresholds are set at the larger of either $15 \%$ of the peak of the emission spectrum in that pixel or a minimum set at $0.04 \mathrm{~K}$. Reducing the $15 \%$ threshold causes little change in the zero- and first-moment maps, but the second-moment map becomes less smooth and has small-scale structure that does not seem to be real based on the spectra themselves. Similarly, reducing the minimum thresholds below 0.04 appears to introduce noise in the second-moment results in areas of low $M_{0}$. 

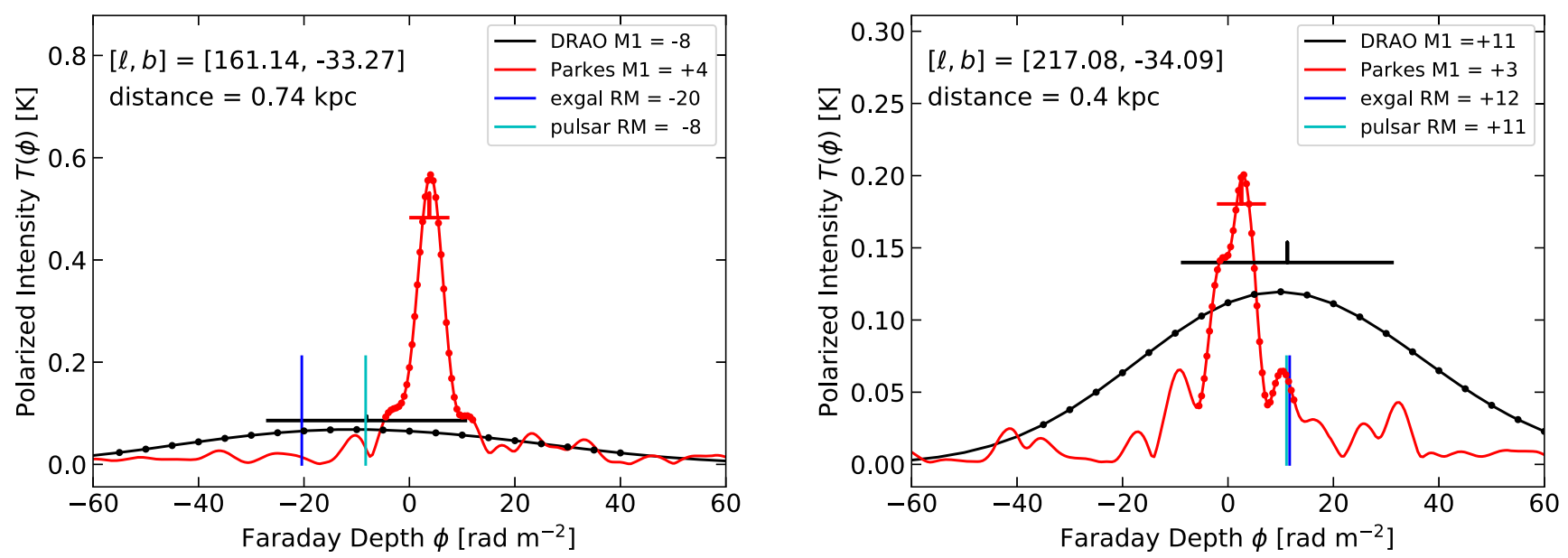

Figure 3. Faraday spectra in the directions of pulsars J0304+1932 (left) and J0452-1759 (right). The colors and symbols are the same as in Figure 2.
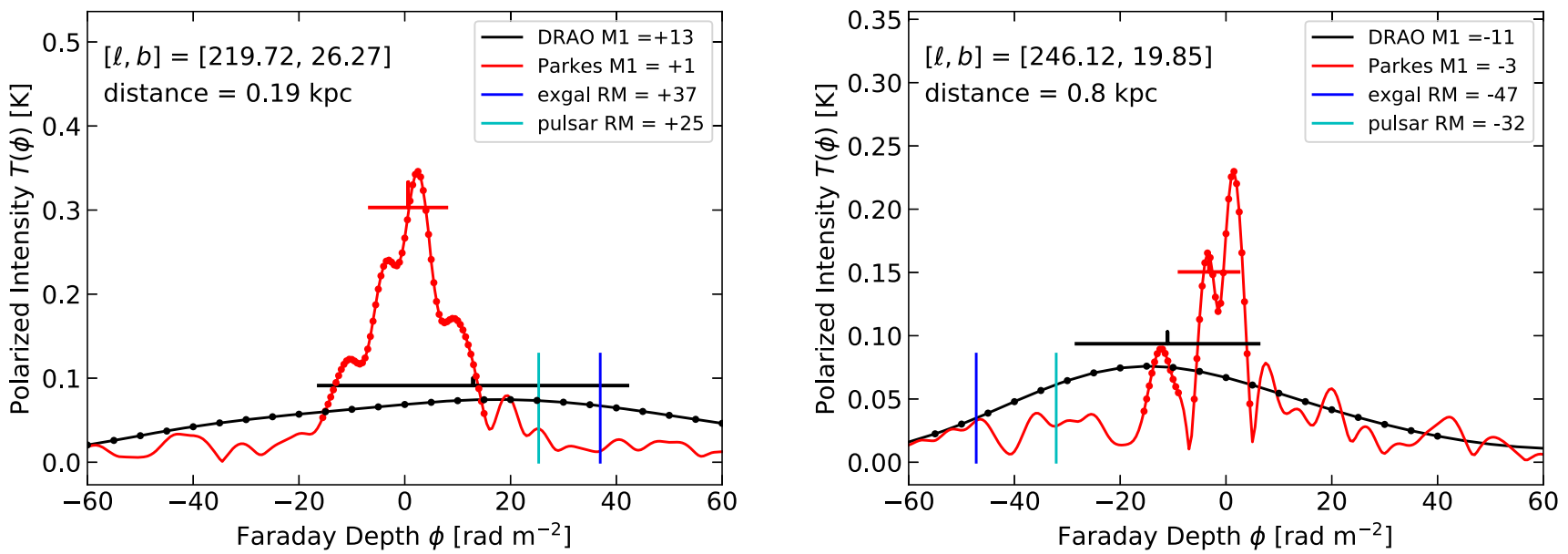

Figure 4. Faraday survey spectra in the direction of pulsars J0837+0610 (left) and J0908-1739 (right). The colors and symbols are the same as in Figure 2.

Channels on either side of the peak are included in the moment calculation until the spectrum drops below the threshold. For the DRAO data, only those channels are used. In some directions, the Parkes spectra show two separate features well above threshold, so we extend the range of channels by fitting a Gaussian to the first feature, then subtracting it from the data, and finding the next peak. If the height of that peak is more than two times the threshold, then we find the range of channels for which $T_{i}$ is above the threshold again. These supplement the channels already selected (from the first peak), and they together make up the channel ranges $i=1 \ldots n$ in Equations (5)-(7). Although this thresholding clearly biases the resulting moments against emission in faint features well separated from the dominant peaks, the moments that result are very consistent with the values of the integral, center, and width of the best-fit Gaussians to each spectrum (see Appendix A). Removing the threshold entirely gives very similar results for $M_{0}$, but the results for $M_{1}$ and $M_{2}$ jump discontinuously from one pixel to another in some areas.

\subsection{Moment Maps}

The zero-moment maps for the Parkes and DRAO surveys are shown in Figure 5. Features in $M_{0}$ for the DRAO survey have good correspondence with known structures, particularly the North Polar Spur (NPS) that reaches from latitude $b \sim 25^{\circ}$ at longitude $\ell \sim 45^{\circ}$ to near the north Galactic pole at $b \sim 75^{\circ}$, where it arches over to $\ell \sim 320^{\circ}$; see Sun et al. (2015) and references therein and Wolleben et al. (2010). Another bright structure in the DRAO $M_{0}$ map is the Fan region near the Galactic plane $\left(b \sim 0^{\circ}\right)$ at longitudes $110^{\circ}<\ell<160^{\circ}$ (Hill et al. 2017). The angular scale of the brightness variations is larger (smoother) at high latitudes and smaller near the Galactic plane. There are also some residual effects of the survey scanning pattern that surround the empty region south of the DRAO decl. limit $\left(\delta=-30^{\circ}\right)$ in the lower right.

In the Parkes $M_{0}$ map much of the NPS and all of the Fan region are north of the decl. limit $\left(\delta=+20^{\circ}\right)$, although there is a hint of a feature aligned with the NPS near $\ell=0^{\circ}$ and $+60^{\circ}<b<+75^{\circ}$. In general, there is very little correspondence between bright regions in the two zeroth-moment maps. The Galactic plane stands out on both, but differently. The plane appears bright in the Parkes map owing to leakage of Stokes $I$ into the Stokes $Q$ and $U$ beams. It is dark on the DRAO map, in part because the leakage has been estimated and subtracted using the low latitudes for calibration $\left(|b|<2^{\circ}\right)$. In the Parkes map there is less of a change in angular scale between high, intermediate, and low latitudes. The lack of correspondence between structures even in the region of 

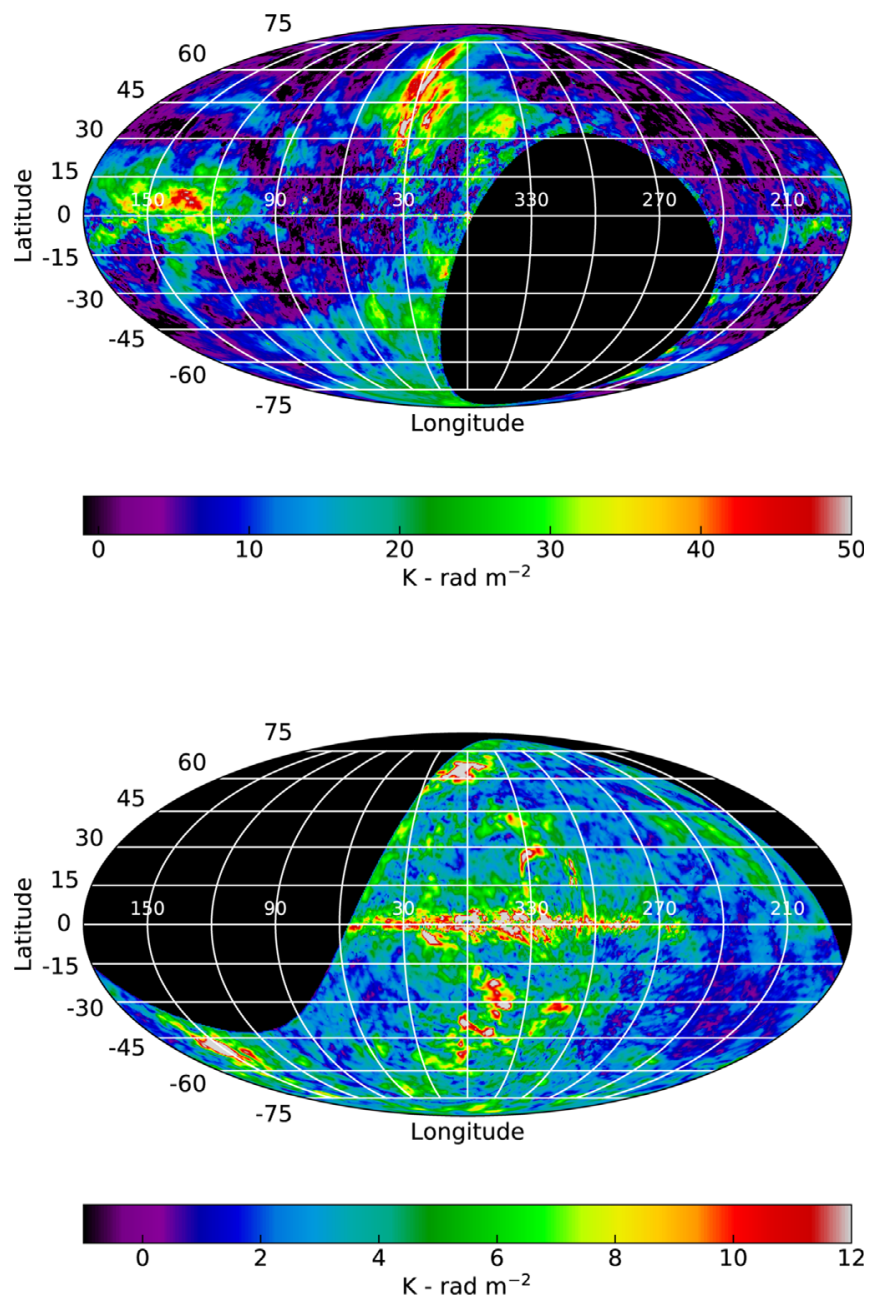

Figure 5. Zero moments $\left(M_{0}\right)$ of the Faraday cubes of the DRAO (top) and Parkes (bottom) surveys, shown in Galactic coordinates and a Mollweide projection. The black areas are either outside the decl. limits of the surveys or positions where the Faraday spectra do not show any features above the minimum threshold of $0.04 \mathrm{~K}$. The units are $\mathrm{K} \mathrm{rad} \mathrm{m}^{-2}$ from Equation (5).

overlap between the two surveys $\left(-30^{\circ}<\delta<+20^{\circ}\right)$ suggests that they are sampling different physical volumes.

The first-moment maps are shown in Figure 6. These show for each pixel the mean of $\phi$ weighted by the brightness temperature. The bright areas around the NPS and the Fan region show quite smooth first-moment values in the DRAO survey with values around $+5 \mathrm{rad} \mathrm{m}^{-2}$, whereas in the Parkes survey the smoothest region is in the fourth quadrant at latitudes $+10^{\circ}<b<+30^{\circ}$. In the Parkes map, the Galactic plane is evident in the first and fourth quadrants with significantly negative $\phi$ compared with most of the rest of the sky. The DRAO cube does not show the Galactic plane very clearly at all, although there is some leakage of bright Stokes $I$ emission into Stokes $Q$ and $U$ in both surveys. Such leakage leads to unreliable values of the moments for both surveys for $|b|<5^{\circ}$.

The second-moment maps (Figure 7) indicate the width of the brightness distribution in $\phi$, similar to the widths of the Gaussians in Figure 1, but now shown for each pixel. In both surveys, the second moment shows a mottled structure, but there is little correspondence between the two.
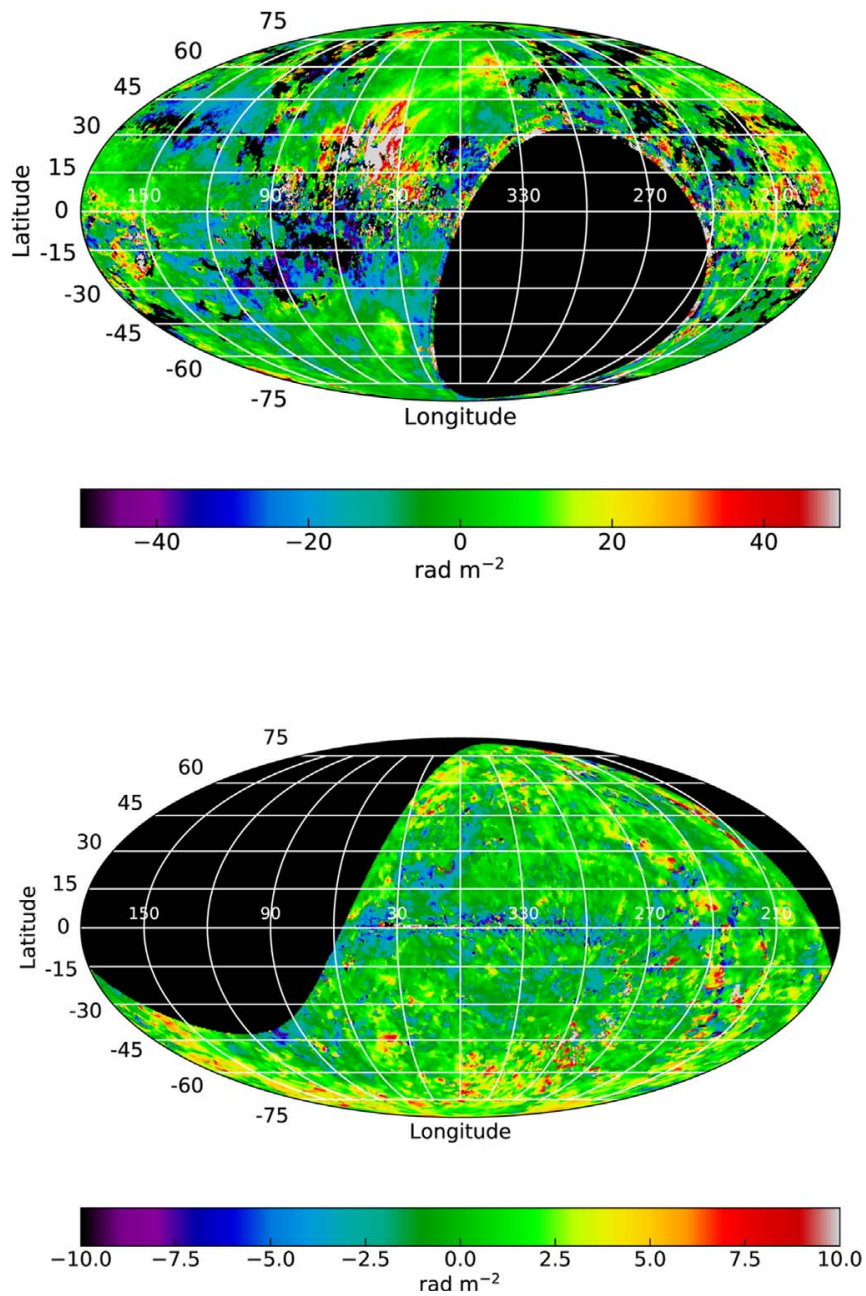

Figure 6. First moments $\left(M_{1}\right)$ of the Faraday cubes of the DRAO (top) and Parkes (bottom) surveys. The units are $\mathrm{rad} \mathrm{m}^{-2}$ from Equation (6). The first moment shows the dominant $\phi$ value at each pixel. Usually this is $\phi$ at the center of the emission in the Faraday spectrum, $F(\phi)$. The black areas are places where the emission is not strong enough to cross the threshold for computation of the moments, or declinations not accessible to the telescopes.

\subsection{Statistics of the First and Second Moments}

To study the statistics of the first and second moments, we take a sample of points separated by $90^{\prime}$ in latitude and in longitude by $90^{\prime} / \cos (|b|)$, i.e., by more than the telescope beamwidths in both surveys. We then separate the samples into sets for different ranges of latitude, $b$. Figures 8-11 show the means and standard deviations of these samples, where the latitude boundaries are set by steps of 0.5 in the cosecant of $|b|$. For a plane-parallel geometry, this is the ratio of the path length through the disk to the scale height of the disk, i.e.,

$$
\operatorname{cosec}(|b|)=\frac{s_{\text {eff }}}{h},
$$

where $h$ is the half-thickness of the plane and $s_{\text {eff }}$ is the path length through the disk at latitude $b$. Here we will not assume a value for $h$, but note that Gaensler et al. (2008) find good evidence that $h \simeq 1.8 \mathrm{kpc}$.

In Figures 8, 9, and 11 the points show the means of distributions of several hundred independent measurements of the moments in the latitude ranges set by the intervals of 

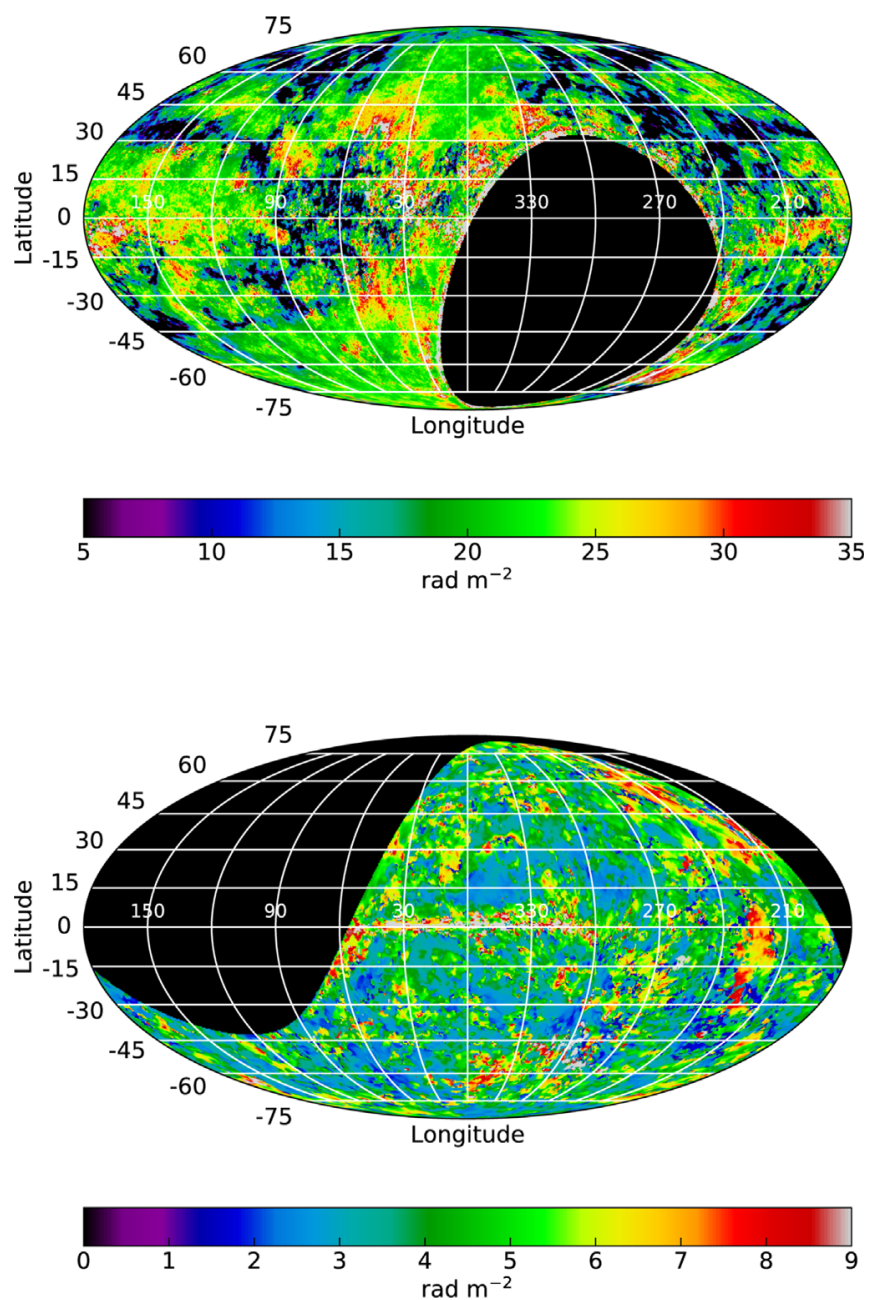

Figure 7. Second moments $\left(m_{2}\right)$ of the Faraday cubes of the DRAO (top) and Parkes (bottom) surveys. The units are $\mathrm{rad} \mathrm{m}^{-2}$ from the square root of Equation (7). The North Polar Spur and the Fan region stand out in all three moments of the DRAO survey. The second moment can be thought of as the width of the emission in Faraday space, similar to the velocity width of a spectral line. For a Gaussian spectral feature, this is just $\sigma_{\phi}$, but if there are several line components, it is the half-width of the range of $\phi$ that they cover.

$\operatorname{cosec}|b|$ on the $x$-axis. The number of points in each sample ranges from $\sim 150$ at the high latitudes to $\sim 1500$ at the lower latitudes. The mean of each sample is plotted as the point, and the standard deviation is plotted as positive and negative bars, without end caps, on each point. The formal error of the mean, calculated simply as the standard deviation divided by the square root of the number of samples, is plotted as the positive and negative error bars with thicker lines and end caps. Thus, although the correlations with $\operatorname{cosec}|b|$ appear to be very weak relative to the longer bars, relative to the errors on the points they are statistically significant. For example, in the DRAO survey in the highest-latitude bin (plotted at 1.25 on the $x$-axis in the left panel of Figure 8) the positive latitude (red) point is $2.26 \pm 0.29 \mathrm{rad} \mathrm{m}^{-2}$, while the negative latitude point is $-1.98 \pm 0.39 \mathrm{rad} \mathrm{m}^{-2}$. The difference is more than 10 times the standard errors. The incomplete coverage of the sky in the two surveys may be a factor in the trends of the moments with latitude. Until the two hemispheres are fully surveyed at both wavelengths, it will be hard to fully characterize the pattern of the local $B$ field, but the averages shown in Figure 8 strongly suggest that there is a $z$ component in the nearby Galactic magnetic field pointing from the northern toward the southern hemisphere.

Looking at the distributions of the first moments versus $\operatorname{cosec}|b|$ in Figure 8, the Parkes points (right panel) show a smooth decrease in moment 1 from positive values at high latitudes (left side) to negative values at intermediate latitudes (right side). The highest value of $\operatorname{cosec}|b|$ shown on the $x$-axis (4.0) corresponds to $|b|=\arcsin 0.25 \simeq 0.25 \mathrm{rad}=14^{\circ}$. Note that both hemispheres show the same trend, i.e., the values are very similar for positive and negative latitudes. Since positive RM corresponds to magnetic fields pointing toward the observer, the implication of the right panel of Figure 8 is that the $B$ field points toward the solar neighborhood at high latitudes in both Galactic hemispheres, but it points away at lower latitudes, in the longitude ranges covered by the Parkes survey (i.e., most of the Galactic southern hemisphere but only about half of the northern hemisphere). If this or some other field geometry is the explanation for the trend in the Parkes survey first-moment points, it is indicated only for the region visible in linear polarization at the Parkes survey wavelength, i.e., close enough to be only weakly depolarized.

The DRAO first-moment points (left panel of Figure 8) show a weak but significant divergence between the two Galactic hemispheres as the path length increases. The positive latitudes shift toward positive $\phi$, thus $\boldsymbol{B}$ pointing toward the Sun, while the negative latitudes shift the opposite way, with $\boldsymbol{B}$ pointing away from the Sun. The two strongest features at latitudes $b>+45^{\circ}$ in the first-moment maps of the DRAO survey (Figure 6, top panel) are the NPS, at longitudes $-30^{\circ}<\ell<+60^{\circ}$, and another smooth feature at longitudes $180^{\circ}<\ell<240^{\circ}$. Both of these show positive values of $M_{1}$, with $3 \mathrm{rad} \mathrm{m}^{-2}<\phi<15 \mathrm{rad} \mathrm{m}^{-2}$. There is very little emission at high positive latitudes that shows negative $M_{1}$. The black lines in Figure 8 indicate the linear regression best fit to all the points in both hemispheres, with the regression coefficients $R=+0.04$ and $R=-0.91$ indicated. Averaging the two hemispheres together, there is almost no correlation of the path length $(\operatorname{cosec}|b|)$ with $M_{1}$ in the DRAO survey, but strong negative correlation between the path length and $M_{1}$ in the Parkes survey.

The Parkes first moment correlated against path length has an $R$ of -0.91 , which indicates a strong anticorrelation between the combined data from the two hemispheres and the path length. The bottom panel of Figure 6 shows more yellow and red (positive first moments) at the highest latitudes in both hemispheres and more dark green and blue at lower latitudes. This shift from positive $M_{1}$ at high latitudes to negative $M_{1}$ at lower latitudes explains the behavior of the latitude averages shown in the right panel of Figure 8. Since negative $\phi$ corresponds to the line-of-sight $B$-field component pointing away from the observer, these two figures suggest two distinct field geometries at high latitudes. The DRAO survey indicates a $B$ field pointing toward the Sun in the north Galactic hemisphere and away from us in the southern hemisphere. On the other hand, in the Parkes survey we see the field pointing toward us from both the Galactic north and south poles, but away from us at intermediate latitudes.

The difference between the DRAO and Parkes second moments is evident in Figure 9. The DRAO widths are much greater than for the Parkes features, 20-25 $\mathrm{rad} \mathrm{m}^{-2}$ compared with $3-5 \mathrm{rad} \mathrm{m}^{-2}$ in the Parkes data. The DRAO survey shows opposite trends in the two Galactic hemispheres: $m_{2}$ increases with path length at positive latitudes but decreases slightly with 

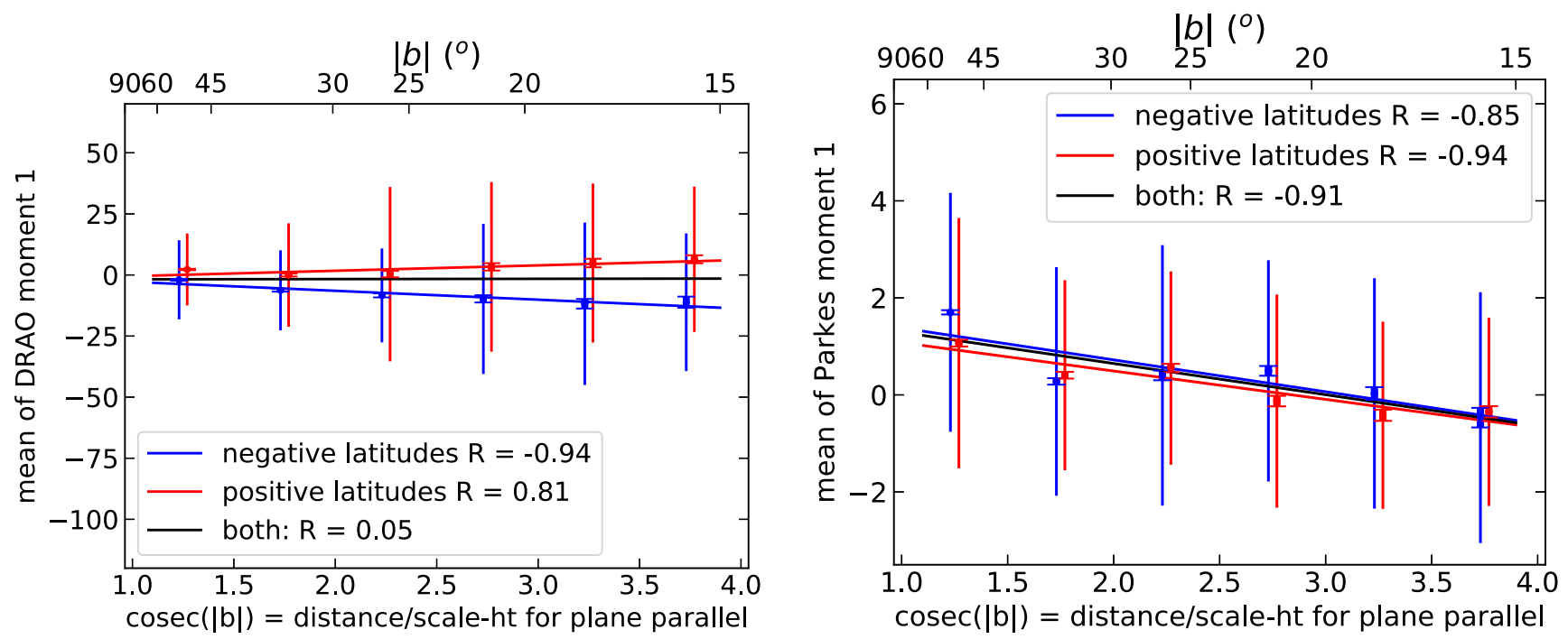

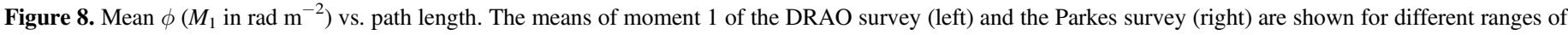

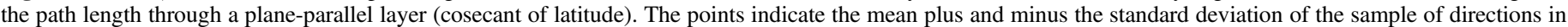

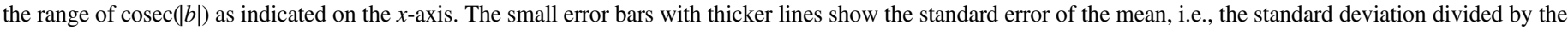
square root of the number of measurements. Values of the correlation coefficients $(R)$ are shown for each linear fit.

path length at negative latitudes. A similar contrary effect is seen in the Parkes data, but it goes the other way around. The strong negative correlation between $m_{2}$ and path length in the Parkes data for positive latitudes suggests that the lower latitudes are not increasing the scatter, as would be expected by increasing the number of steps in a random walk process of field reversals. This, in turn, suggests that the polarization horizon is so nearby for the emission seen in the Parkes survey that the local interstellar medium, including the Local Bubble (Frisch et al. 2012; Alves et al. 2018), is dominating the width of features in the Faraday spectra. In the Parkes data the negative latitudes show a weak positive correlation between $m_{2}$ and path length. A similar horizon effect in M51 might explain the difference in the width of $T(\phi)$ measured at $1-2 \mathrm{GHz}$ compared with that measured at 5-8 GHz (Mao et al. 2015b).

\section{Comparison with Other RM Surveys}

The Faraday spectra of the diffuse polarized emission illustrated in the previous section can be compared with other tracers of the RM at high and intermediate latitudes. The most comprehensive is a compendium of surveys of extragalactic radio source RMs compiled and gridded by Oppermann et al. (2012, 2015). We have made comparisons with both the 2012 and 2015 versions of the Galactic foreground RM maps of Oppermann et al., as the former is more directly derived from the data, while the latter is based on models that best reproduce the data. In comparison with the GMIMS survey results, the two give similar information. Below we use the 2015 map ("maps/phi" available from https://wwwmpa.mpa-garching. mpg.de/ift/faraday/2014/index.html). The contribution of the Milky Way foreground derived from the Oppermann model is shown in Figure 10. Although this is the estimate for the Galactic foreground, we will refer to it as the "extragalactic RM grid" or just the "extragalactic RMs."

Sampling the extragalactic RMs at the same points as for Figures 8 and 9 gives Figure 11. The RM values on the $y$-axis of Figure 11 and on the scale of Figure 10 are much larger than the range of $\phi$ with bright emission in the Parkes survey. The width of the distribution of RMs (vertical bars) increases rapidly with $\operatorname{cosec}|b|$, as expected for a random walk process where the line of sight passes through many uncorrelated regions where the $B$-field component is sometimes toward the observer (positive $\phi$ ), sometimes away (negative), coupled with the higher average density of the ionized medium at low $|z|$ and higher $B$-field intensity at low $|z|$. This is similar to the increase in the DRAO first moments, $M_{1}$, with increasing cosec $|b|$ shown in the left panel of Figure 8. In addition, the extragalactic RM means (red and blue points in Figure 11) separate to positive and negative values for the northern and southern hemisphere samples, in the same way that the DRAO first-moment points do in the left panel of Figure 8. The consistency of this effect suggests that it is caused by the largescale ordered $\boldsymbol{B}$ field similar to that seen at low latitudes in surveys of RMs toward compact sources (e.g., Mao et al. 2012; Han 2017; Ordog et al. 2017). In both Figure 10 and the top panel of Figure 6, the overall picture for the inner Galaxy is positive RMs at positive latitudes $\left(0^{\circ}<b<30^{\circ}\right)$ in the first quadrant, negative RMs at negative latitudes in the first quadrant, and the opposite in the fourth quadrant. The larger absolute numbers in the extragalactic sample are expected based on the factor of two between the peak $\phi$ measured for the emission from a slab and the RM seen toward a source behind the slab. The implication is that the DRAO first moments are tracing roughly the same ordered field component as traced by the extragalactic and pulsar RMs, whereas the Parkes first moments are tracing something quite different (see Han 2017, Figure 6).

The correlations with distance that appear in Figures 8 and 9 are all the more interesting considering that the moments are not correlated with each other, especially at low latitudes. The first moments of the two surveys are compared with the extragalactic foreground sample in Figure 12.

\subsection{Pulsar RMs}

RMs have been determined for 1001 pulsars (Manchester et al. 2005 version 1.56 supplemented by Han et al. 2018b; see 

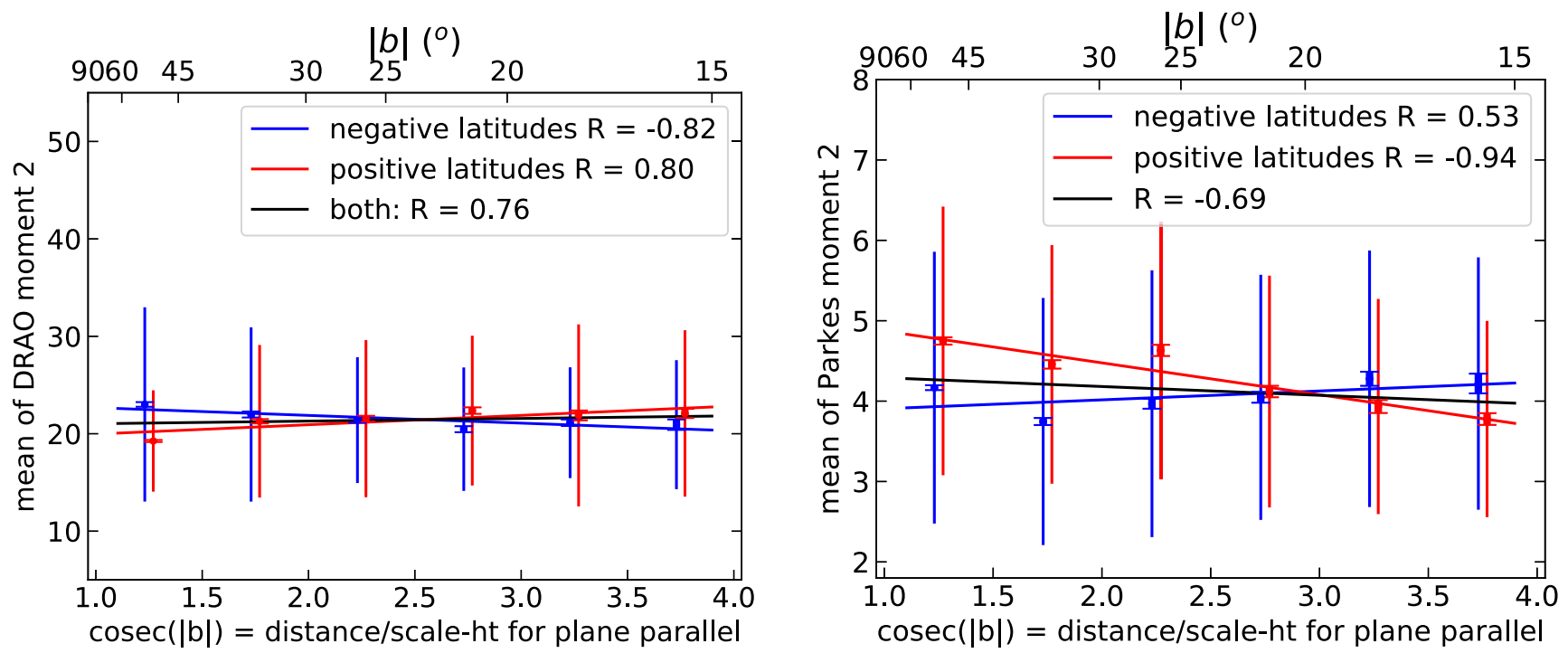

Figure 9. $\phi$ width $\left(m_{2}\right.$ in rad $\left.\mathrm{m}^{-2}\right)$ vs. path length. The means of moment 2 of the DRAO survey (left) and the Parkes survey (right) are shown for ranges of cosecant $(|b|)$. The points indicate the mean plus and minus the standard deviation, with the thicker error bars indicating the standard error of the mean, as in Figure 8 .
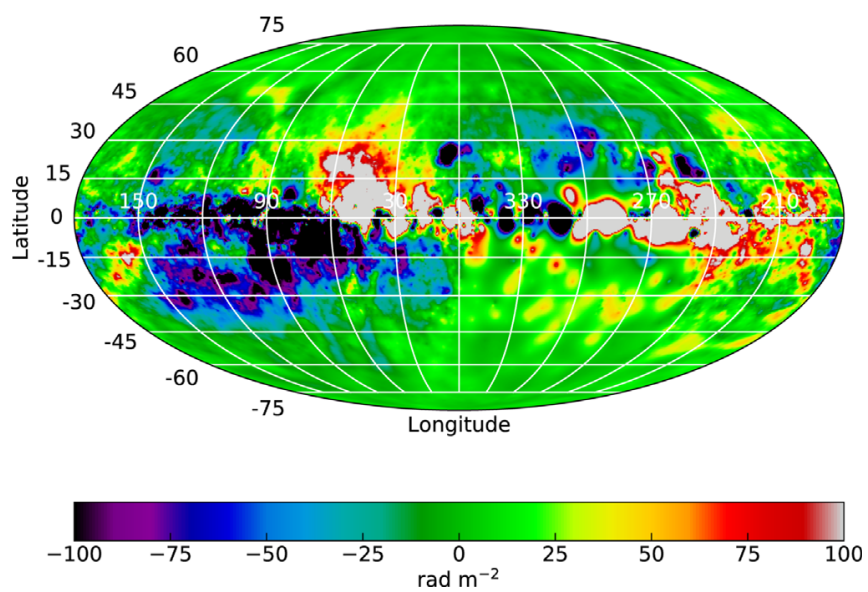

Figure 10. Foreground Galactic RM grid from Oppermann et al. (2015) projected on the same coordinates as Figures 5-7. At low latitudes $\left(|b|<5^{\circ}\right)$ the scale is saturated. There the extremes are $-1124 \mathrm{rad} \mathrm{m}^{-2}<\phi<1273 \mathrm{rad} \mathrm{m}^{-2}$.

Han et al. 2018a); most of these also have distance determinations, from either dispersion, parallax, or other means. Although the RM and dispersion measure (DM) are not physically independent, since both involve the line-of-sight integral of the electron density, observationally they constitute entirely separate measurements. Most of the pulsar distances are based on combining DM values with an electron density model of the Milky Way. Although this does not give a very precise distance, it is in some ways just what we want for comparison of the pulsar and diffuse RMs, since we might expect more RM, or more fluctuation in RM, on a path with higher DM, i.e., a higher path integral of electron density. Hence, we will make use of the pulsar distances and RMs as milestones to compare with the $\phi$ distribution of the diffuse polarized emission, keeping in mind that most individual pulsar distances are not reliable to better than about $30 \%$ at latitudes $|b|>20^{\circ}$.

Note that pulsars, like the extragalactic sources that have been used to form the Oppermann extragalactic RM grid,

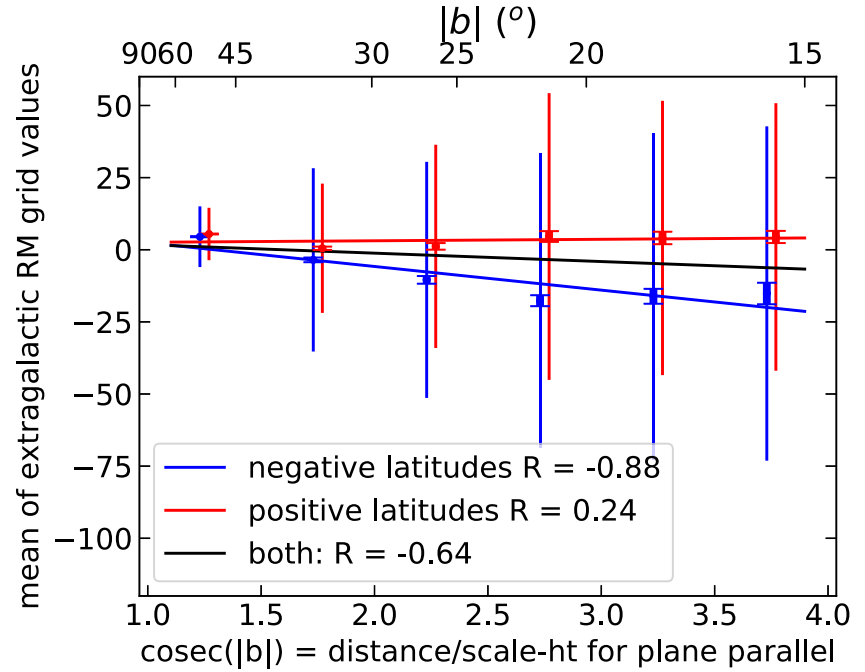

Figure 11. Extragalactic RM grid, sampled at the same points that are used for Figures 8 and 9, data from Oppermann et al. (2015). The gradual separation of the red and blue points at lower latitudes, similar to that seen in the left panel of Figure 8, suggests that the north Galactic hemisphere has a net line-of-sight $\boldsymbol{B}$-field component pointing toward the Sun, while the southern hemisphere has a net $\boldsymbol{B}$-field component pointing away from the Sun. The leftmost points are at $4.52 \pm 0.19 \mathrm{rad} \mathrm{m}^{-2}$ in blue (negative latitudes) and $5.45 \pm 0.16 \mathrm{rad} \mathrm{m}^{-2}$ in red (positive latitudes), a $5 \sigma$ difference.

are all compact sources, so they do not suffer depolarization due to the Galactic magneto-ionic medium. But the pulsar distances are often much less than the path length entirely through the ionized interstellar medium (Reynolds layer) that can cause Faraday rotation and depolarization of the diffuse emission. Thus, pulsar RMs are not perfectly correlated with the extragalactic RMs. This is particularly true when the large number of pulsars at low latitudes are included in the sample, as shown in the left panel of Figure 13. On the other hand, for pulsars at latitudes above $|b|=25^{\circ}$ and distances less than a few kiloparsecs, the correlation is better $(R=0.75$; right panel of Figure 13). 

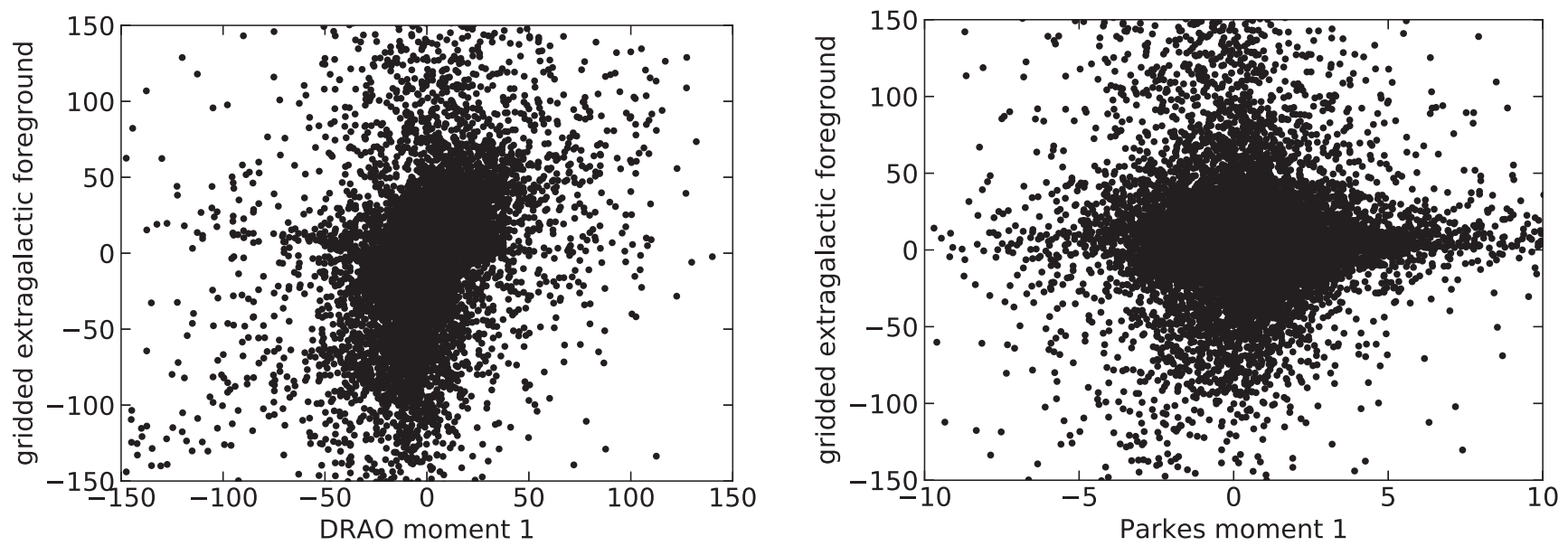

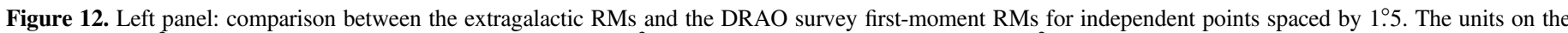

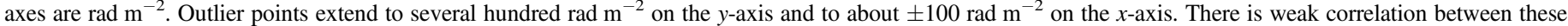

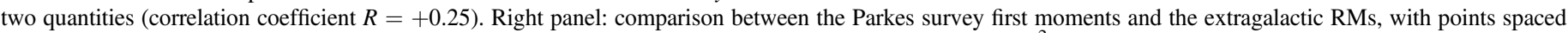

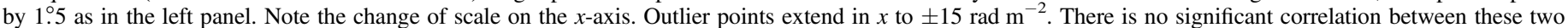
quantities (correlation coefficient $R=-0.02$ ).

\section{Distances}

Comparison of the pulsar RMs to the first moments of the polarization surveys in the directions of the pulsars is useful to see roughly the range of distances from which the bulk of the polarized emission must come. Figure 14 shows moderate correlation between pulsar RMs and first moments in the DRAO survey, with the pulsars selected to be at latitudes above $|b|=25^{\circ}$ and with distances $d<5 \mathrm{kpc}$ (right panel). The correlation is better if we restrict the distances of the pulsars to $d<700 \mathrm{pc}$, as shown in the left panel of Figure 14. Although the number of points is less $(n=13)$, the correlation coefficient is higher, $R=0.75$ versus $R=0.41$ for the larger sample. The probability of null hypothesis, i.e., the chance that the sample is taken from a population with $R=0$, formally the "two-sided $P$ value," is 0.0032 for the left panel ( $n=13$ points) and 0.0023 for the right panel ( $n=52$ points) of Figure 14 .

In Table 3 are shown the $R$ and $P$ values for samples of pulsars selected by distance $\left(d<d_{\max }\right)$. The first moments of the DRAO and Parkes surveys and the Galactic foreground computed from the extragalactic grid of RMs are correlated against the RMs of the pulsars in the same directions. The effects illustrated in Figures 13 and 14 are similar for many of the samples in the table. However, although the extragalactic RMs are correlated with the pulsar RMs with $R$ between 0.69 and 0.88 for the full range of distances, the DRAO first moments show stronger correlation with pulsar RMs for samples with distances less than about $1.5 \mathrm{kpc}$, and the strongest correlation is for $d<700 \mathrm{pc}$. Note that the numbers of pulsars in each sample and their $\sigma_{\mathrm{RM}}$ values, shown in the second and third columns of Table 3, are computed over the whole sky. The numbers of pulsars in the areas of the DRAO and Parkes surveys are smaller, indicated by $n$ in Columns (5) and (8) of the table.

For the Parkes survey first moments, there is negative correlation with the pulsar RMs for similar samples of nearby, high-latitude pulsars (e.g., $R=-0.38$ for a sample of 15 pulsars in the Parkes decl. range with distance less than $0.7 \mathrm{kpc}$ ). This anticorrelation is not statistically significant $(P=0.16)$. Similarly, there is no significant correlation between the Parkes first moments and the extragalactic RMs in the directions of nearby pulsars. The absence of correlations between the Parkes first moments and other RM tracers suggests that the high-latitude polarized emission seen at the low frequencies of the Parkes survey is mostly quite nearby, probably within a few hundred parsecs. There may be more distant emission in some areas; discrete structures at greater distances would be missed by these small samples of pulsar-selected directions.

A rough idea of the distance to the bulk of the Parkes survey polarized emission is indicated by Figure 15. Selecting pulsars with $|b|>25^{\circ}$ as in the analysis above, the standard deviation of the pulsar RMs increases with the distance of the sample, starting from about $\sigma_{\mathrm{RM}}=12 \mathrm{rad} \mathrm{m}^{-2}$ for a distance of about $200 \mathrm{pc}$ and increasing smoothly to $26 \mathrm{rad} \mathrm{m}^{-2}$ for a distance of $1.6 \mathrm{kpc}$ (in bins of width a factor of two in distance). The dispersion of the all-sky average Faraday spectrum of the Parkes survey data is just $4.5 \mathrm{rad} \mathrm{m}^{-2}$ (Table 2 and Figure 1). From this we draw the conclusion that the bulk of the polarized emission at $300-500 \mathrm{MHz}$ is coming from distance less than $0.3-0.5 \mathrm{kpc}$ if we assume that the scatter of the pulsar RMs is generated by the same process as the width of the Faraday spectrum of the polarized emission, and bearing in mind that a background source should show twice the mean RM of a slab that has mixed emission and Faraday rotating material. At high latitudes, most of the scatter in both quantities probably comes from a random walk through the line-of-sight distribution of magnetic field directions and interstellar electron densities. This process leads to the depolarization of the emission from distances greater than about $1 \mathrm{kpc}$.

\section{Faraday Depolarization}

Although the RM does not increase monotonically with distance along the line of sight, the Faraday depolarization does. Thus, the polarization horizon at any given wavelength may recede or approach the observer by factors of three or even 10 from one direction to another. Several different physical processes contribute to depolarization, falling into four groups: depth depolarization, beam depolarization, bandwidth depolarization, and geometric depolarization (Burn 1966; Tribble 1991; Sokoloff et al. 1998). Bandwidth depolarization depends on the resolution of the spectrometer, as given in Table $1 \delta \phi, \phi_{\max }$, and $\phi_{\text {max-scale. }}$ These depend on the survey parameters and on 

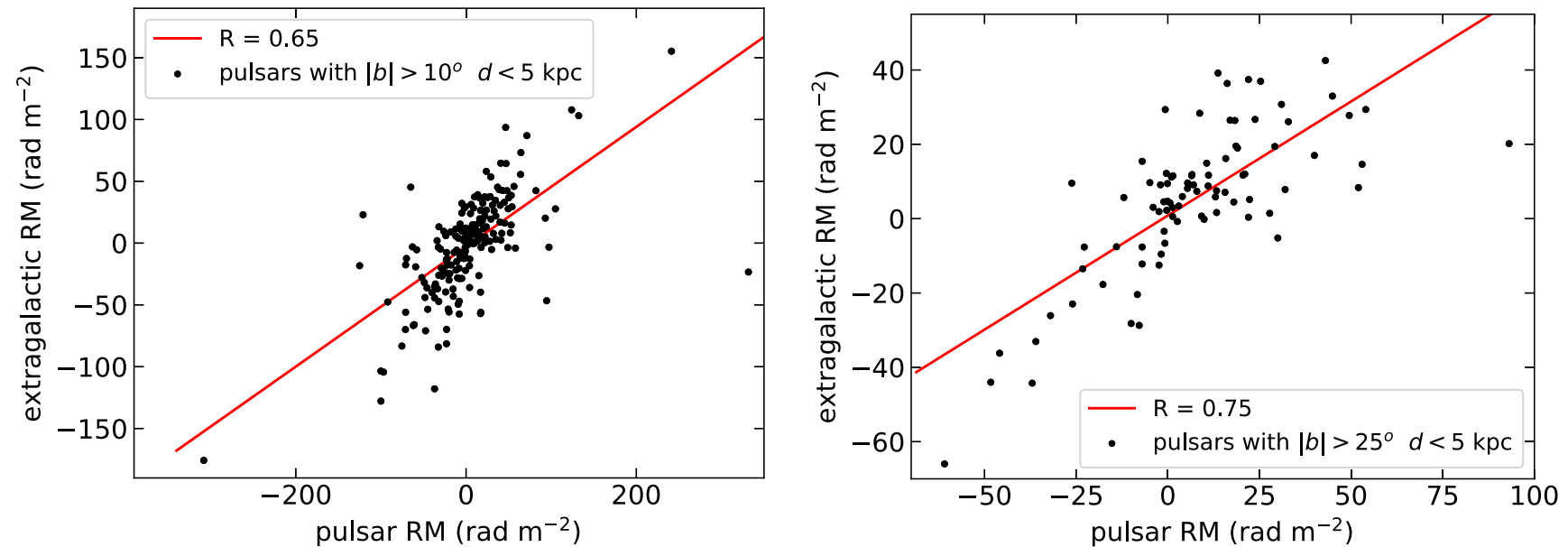

Figure 13. Pulsar RMs compared with the extragalactic foreground RM at the same positions. Including all pulsars with $|b|>10^{\circ}$ and distance $d<5 \mathrm{kpc}$, there is significant correlation $\left(R=0.65\right.$; left panel), but there is stronger correlation for pulsars above $|b|=25^{\circ}$ with distances less than $5 \mathrm{kpc}(R=0.75$; right panel). Including pulsars with latitudes below $|b|=5^{\circ}$ washes out the correlation with the extragalactic foreground. The red lines are least-squares linear fits to the data points, with correlation coefficients $R$ as indicated.
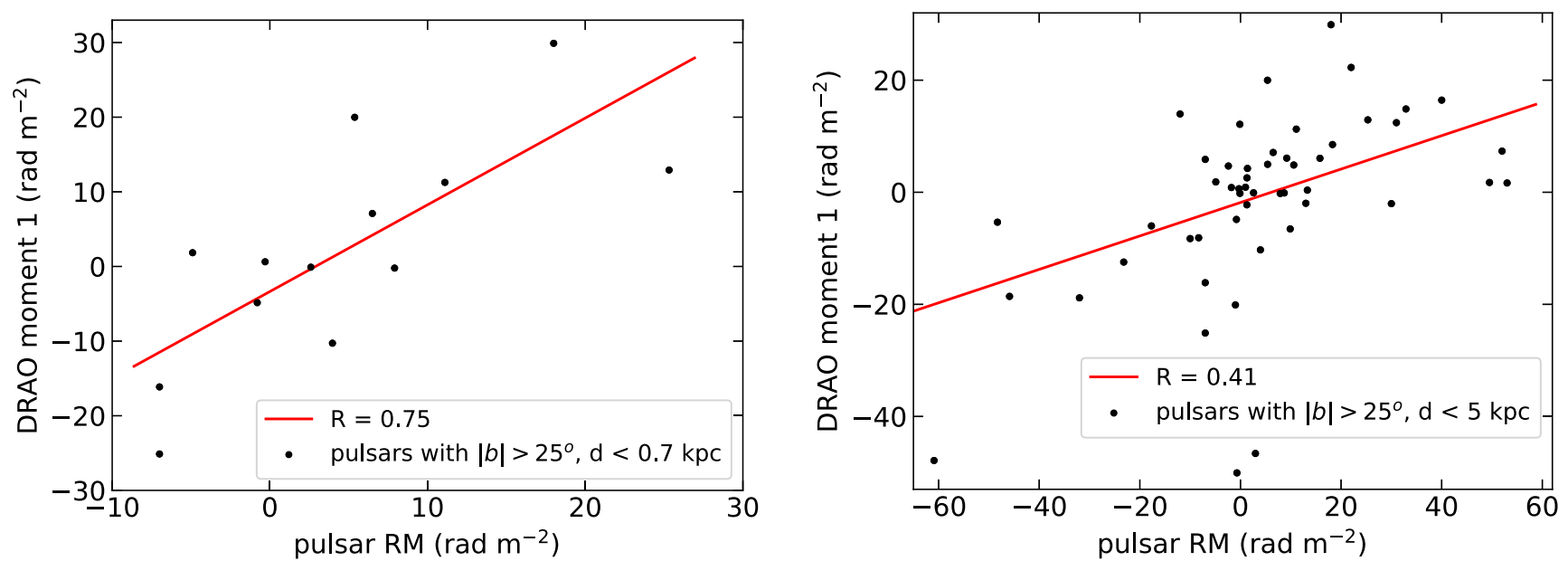

Figure 14. DRAO survey first moments in the directions of pulsars with known RMs. Left panel: 13 pulsars above $|b|=25^{\circ}$ with distance $d<0.7 \mathrm{kpc}$. Right panel: 53 pulsars above $|b|=25^{\circ}$ with distance $d<5 \mathrm{kpc}$. The smaller sample of the more nearby pulsars shows considerably better correlation than the larger sample $(R=0.75$ vs. $R=0.41)$. They both have quite low $P$ values $(0.0032$ and 0.0023 ; see Table 3$)$, indicating that the probability of null hypothesis is well below $1 \%$.

Table 3

Correlation Coefficients-Pulsars with $|b|>25^{\circ}$

\begin{tabular}{|c|c|c|c|c|c|c|c|c|c|c|}
\hline \multicolumn{3}{|c|}{ Pulsar Sample } & \multicolumn{3}{|c|}{ DRAO Moment 1} & \multicolumn{3}{|c|}{ Parkes Moment 1} & \multicolumn{2}{|c|}{ Extragalactic } \\
\hline$\overline{d(\mathrm{kpc})}$ & $\bar{n}$ & $\overline{\sigma_{\mathrm{RM}}}$ & $\bar{R}$ & $\bar{n}$ & $\bar{P}$ & $R$ & $n$ & $\bar{P}$ & $\bar{R}$ & $P$ \\
\hline$d<0.3$ & 8 & 11.6 & 0.74 & 5 & 0.15 & -0.17 & 7 & 0.70 & 0.75 & 0.033 \\
\hline$d<0.7$ & 19 & 13.2 & 0.75 & 13 & 0.0032 & -0.38 & 15 & 0.16 & 0.88 & $1 \times 10^{-6}$ \\
\hline$d<1.0$ & 34 & 15.6 & 0.45 & 22 & 0.036 & -0.36 & 26 & 0.08 & 0.73 & $1 \times 10^{-6}$ \\
\hline$d<1.5$ & 54 & 16.8 & 0.42 & 33 & 0.014 & -0.21 & 44 & 0.18 & 0.69 & $<10^{-6}$ \\
\hline$d<2.0$ & 65 & 19.8 & 0.27 & 40 & 0.09 & -0.26 & 53 & 0.07 & 0.74 & $<10^{-6}$ \\
\hline
\end{tabular}

the Faraday depth. For high values of $\phi$, close to $\phi_{\max }$, the finite channel width attenuates the strength of the polarized signal. Depth depolarization is a radiative transfer effect in a medium with mixed thermal and cosmic-ray electrons and magnetic field, where Faraday rotation changes the plane of polarization of the radiation as it moves toward the observer along the line of sight. After propagating through a medium for a distance such that $\chi \simeq \pi \mathrm{rad}$, the polarization from the near side destructively interferes with that from the far side. The distance required is inversely proportional to $\lambda^{2}$, by Equation (1). This occurs even in an entirely uniform medium, but also in a medium with irregularities in the electron density and/or the 


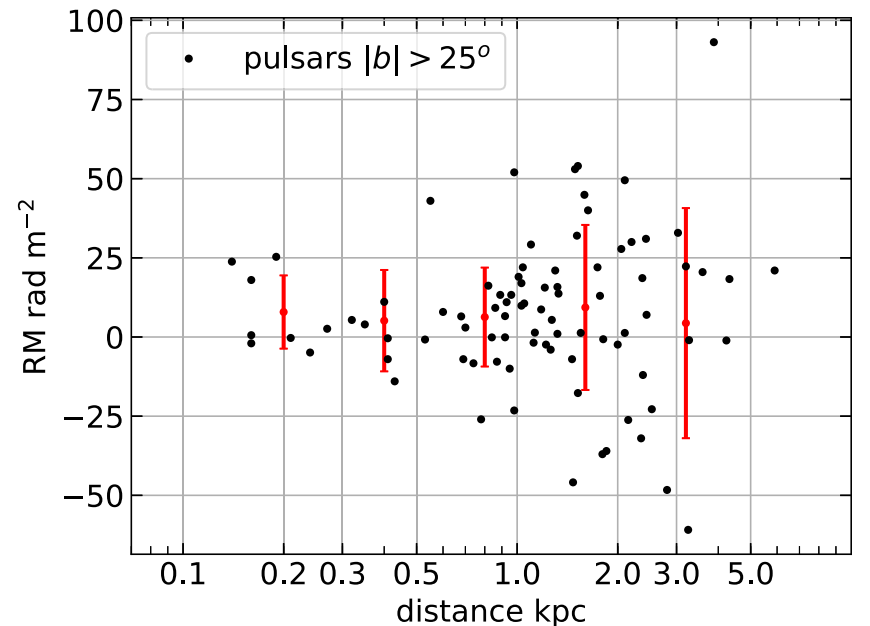

Figure 15. Distribution of pulsar RMs vs. distance on a semi-log scale. The dispersion of the RM distribution increases with distance, as indicated in Column (3) of Table 3 and shown by the red error bars.

strength or direction of the $\boldsymbol{B}$ field. Geometric depolarization occurs when two emission regions along the same line of sight have different projections of the $\boldsymbol{B}$ field on the plane of the sky, so that their polarization adds in a random way, and Stokes $Q$ or $U$ or both can sum to zero. Geometric depolarization is not a Faraday effect (it is independent of $\lambda$ ), but its effect can be mixed with Faraday rotation to give a $\lambda$ dependence. Finally, beam depolarization comes from variation of the position angle of the linear polarization on different lines of sight within the area of the telescope beam, caused either by the geometry of the emission or by changes in the Faraday depth along nearby lines of sight, which are not resolved by the telescope. For the single-dish observations described here, with beamwidths of

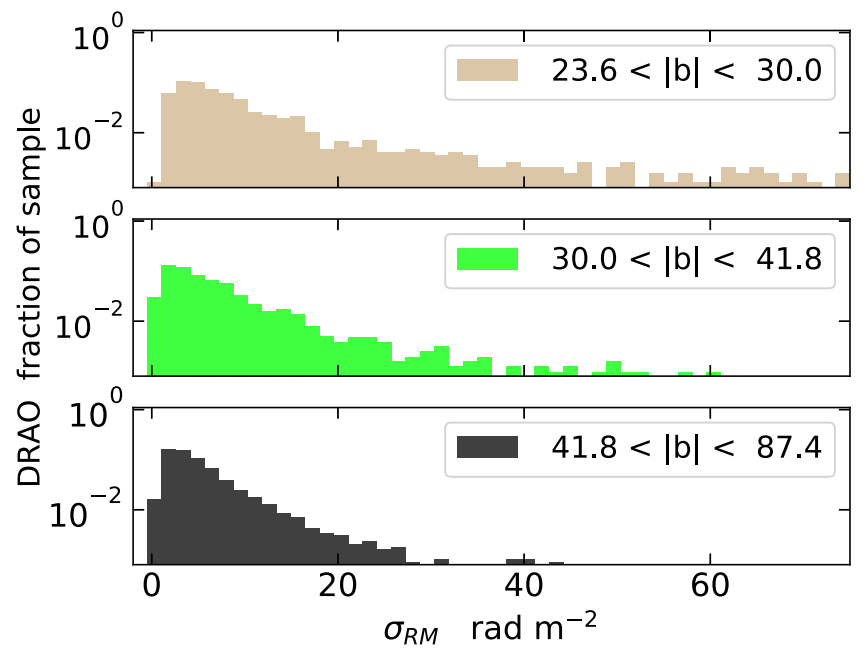

Figure 16. Distribution of standard deviations of the first moments, $\sigma\left(M_{1}\right)$, for the DRAO survey. The values of $\sigma$ are computed for each point in a grid of centers separated by $90^{\prime}$, with $\sigma$ the standard deviation of the values of the first moment for each pointing center, in an annulus of points between 58' and 68' (Equation (9)) from the center. Samples of $\sigma$ values measured in different latitude ranges, corresponding to steps of 0.5 in $\operatorname{cosec}|b|$, are shown separately. Note that the $y$ axis is logarithmic and the distributions decrease roughly exponentially above their peaks (linear on the semilogarithmic axes of these figures).

Burn 1966, Equation (18)). Taking $A$ and $C$ as the real and imaginary parts of $S$ in Equation (8) gives

$$
\begin{aligned}
\mathrm{DP} \approx & \mid \frac{1}{A^{2}+C^{2}}\left\{A-e^{-A}(A \cos C-C \sin C)\right. \\
& \left.-i\left[C-e^{-A}(A \sin C+C \cos C)\right]\right\} \mid
\end{aligned}
$$

and finally

$$
\mathrm{DP} \approx \frac{1}{A^{2}+C^{2}} \sqrt{\left[A-e^{-A}(A \cos C-C \sin C)\right]^{2}+\left[C-e^{-A}(A \sin C+C \cos C)\right]^{2}}
$$

$30^{\prime}-80^{\prime}$, beam depolarization and depth depolarization are the most significant effects that limit the distance that these surveys can see.

For the simplified case of beam depolarization arising from varying Faraday rotation mixed inhomogeneously with polarized emission along different lines of sight within the telescope beam, Sokoloff et al. (1998) derive a result (their Equation (34)) for the combined effects of depth and beam depolarization, DP, based on a single complex parameter, $S$. If $T\left(\lambda^{2}\right)$ is the observed polarized intensity (Equation (2)) and $T_{0}\left(\lambda^{2}\right)$ is the intrinsic polarized brightness of the source, then

$$
\mathrm{DP} \equiv \frac{T}{T_{0}} \approx\left|\frac{1-e^{-S}}{S}\right|
$$

where the parameter $S$ is defined as

$$
S=2 \sigma_{\mathrm{RM}}^{2} \lambda^{4}-2 i \lambda^{2} \mathbb{R}=A+i C,
$$

where $\mathbb{R}$ is the (maximum) Faraday depth of the emission region, which we take to be equal to the absolute value of the extragalactic foreground RM, and $\sigma_{\mathrm{RM}}$ is the rms fluctuation of the RM measured on the scale of the beamwidth (see also where $A$ increases as $\lambda^{4}$ and $C$ increases as $\lambda^{2}$ as we go to longer wavelengths. Thus, Equation (9) is consistent with the conclusion of Tribble (1991) that DP $\propto \lambda^{4}$ at short wavelengths and DP $\propto \lambda^{2}$ at long wavelengths.

To evaluate $\sigma_{\mathrm{RM}}$ for the two GMIMS surveys, we compute the standard deviation of the observed first moments over an annulus just outside the $40^{\prime}$ beam radius of the Parkes survey. For each independent pointing center, i.e., pixels spaced by $90^{\prime}$ in latitude and in longitude $/ \cos (|b|)$, we take the standard deviation over all pixels $i=1 \ldots N$ that are in an anulus with inner radius $58^{\prime}$ and outer radius $68^{\prime}$ :

$$
\sigma_{M 1}=\sqrt{\frac{1}{N} \sum_{i=1}^{N}\left(M_{1, i}-\bar{M}_{1}\right)^{2}}
$$

(note that the NumPy "nanstd" function used here gives the population standard deviation rather than the sample standard deviation, which has $N-1$ in the denominator instead of $N$ ). The number of pixels $N$ contributing to these samples depends on the latitude, but it is typically 12 or more. The distribution of values of $\sigma$ determined for these annuli for the two surveys is shown in Figures 16-18. Also shown are $\sigma$ values computed 


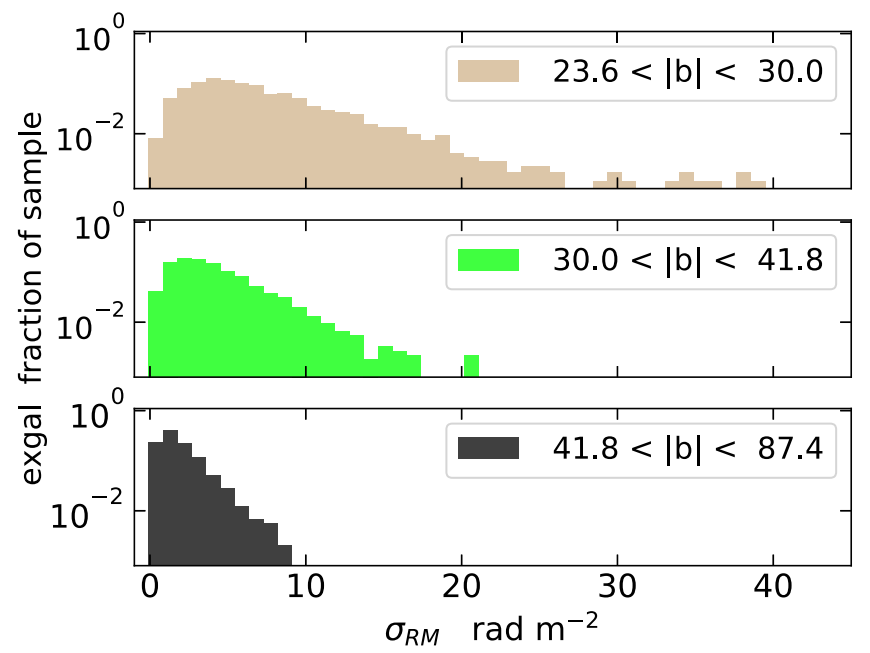

Figure 17. Distribution of standard deviations of RMs, $\sigma(\mathrm{RM})$, computed for the extragalactic foreground RMs. The center points and annular areas are the same as used for Figure 16 and 18, as are the axes on the figure, but note the different scale on the $x$-axis.

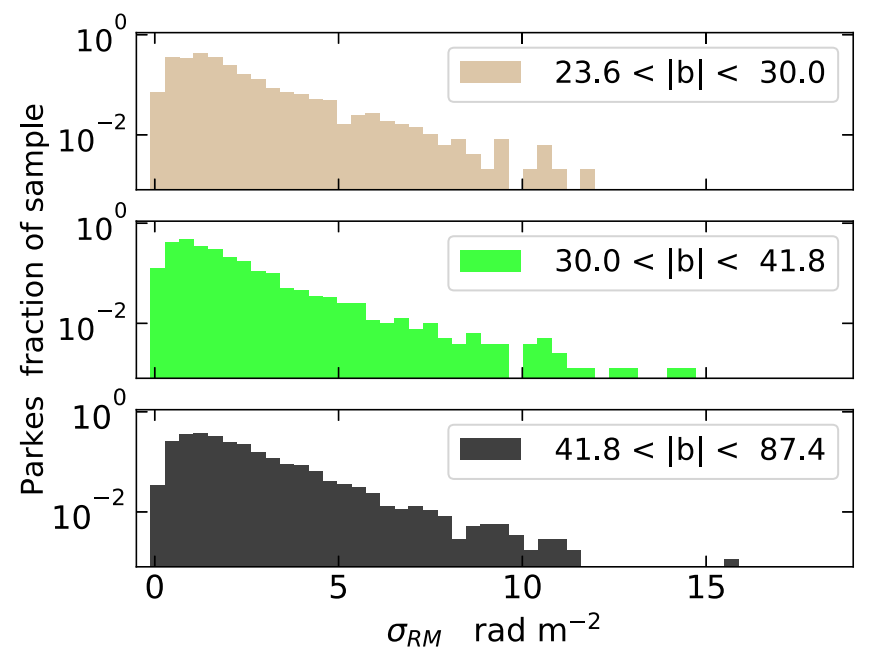

Figure 18. Distribution of standard deviations of RMs, $\sigma\left(M_{1}\right)$, computed for the Parkes survey first moments. The center points and annular areas are the same as used for Figure 16, as are the axes on the figure, but note the different scale on the $x$-axis.

for the extragalactic sample of RMs, over the same areas with the same centers. The progressively narrower distribution of RMs at higher latitudes was noted by Schnitzeler (2010), using a similar $\operatorname{cosec}|b|$ approach to separate the Galactic and extragalactic contributions to the RMs of NVSS sources. Here the averaging associated with the Oppermann et al. (2015) model separates the Galactic foreground from the extragalactic RM contribution, at least nominally.

Although $\sigma_{M 1}$ is computed over areas on the sky about two to four times larger than the beamwidth of the telescope, we will assume that it gives an estimate, probably an overestimate, of $\sigma_{\mathrm{RM}}$, which is the rms fluctuation of the RM in a single beam area. We cannot measure $\sigma_{\mathrm{RM}}$ inside the DRAO and Parkes beams without going to higher resolution, with either a larger single dish or an aperture synthesis telescope. For the simplified analysis in this section, we will assume that the two are roughly equal. Using these values of $\sigma_{\mathrm{RM}} \simeq \sigma_{M 1}$ measured over the annular areas around each of the grids of pointing centers, we can determine the expected depolarization using Equations (8) and (9). These are shown in Figure 19 for three ranges of Galactic latitude, with $\operatorname{cosec}|b|$ in the ranges $1-1.5,1.5-2$, and $2-2.5$. For the Parkes data (left panel), the values of DP are mostly less than $10^{-1}$, with some below $10^{-2}$ at the lower latitudes. On the other hand, for the DRAO survey, the median value of DP predicted for latitudes above $|b|=42^{\circ}$ is 0.77 . Thus, depolarization should not be very significant for this survey at high latitudes. At lower latitudes the medians decrease to 0.53 and 0.29 in the middle and top right panels of Figure 19, respectively. Hence, depolarization is becoming significant at intermediate latitudes. This result explains why the correlation between $M_{1}$ from the DRAO survey and the pulsar RMs weakens for pulsar distances greater than $700 \mathrm{pc}-$ $1 \mathrm{kpc}$. For example, if the median DP $=0.5$ at $\operatorname{cosec}|b|=2$ $\left(b=30^{\circ}\right)$ and this corresponds to a distance of 800-1000 pc, then the scale height of the magneto-ionic layer causing the depolarization at this wavelength should be about 400-500 pc. Although the depolarization estimates derived from Equations (7) and (8) appear to be conclusive in explaining the difference between the Parkes and DRAO survey volumes, these equations were derived for an idealized situation more relevant to supernova remnants or other galaxies than to the allsky surveys discussed here. More analysis and simulations will give a better understanding of the wavelength dependence of the Faraday depolarization.

\section{Conclusion}

Our Galaxy presents many faces; various tracers of the interstellar medium show the effects of the many different physical processes at work. The magnetic field shapes the features of these faces, even for the spectral line tracers of the cool neutral medium and the molecular medium (e.g., Clark 2018; Zamora-Avilès et al. 2018). For the ionized medium and the cosmic-ray electrons, the magnetic field is an important and often dominant factor in their dynamics and evolution. The polarization of the diffuse synchrotron emission observed at high frequencies (Page et al. 2007; Miville-Deschênes et al. 2008) shows the structure of the magnetic fields and the cosmic-ray electrons that fill the disk and extend into the halo. At lower frequencies, the Faraday spectrum of the Galactic diffuse synchrotron emission shows the juxtaposition of the emission regions with the diffuse ionized medium that causes the Faraday rotation. The Faraday rotating medium is thermal plasma, again with a magnetic field, although this time it is the line-of-sight component of the field that matters, in contrast to the component in the plane of the sky that determines the position angle of the polarized emission. Thus, the Faraday spectrum holds the promise of providing distance information; someday it may be one of several observational techniques that will allow an accurate 3D model of the Galaxy to be constructed including the magnetic field, the cosmic-ray electrons, and the diffuse ionized medium (Su et al. 2018). This goal overlaps that of much recent work by low-frequency arrays such as LOFAR and the MWA (Iacobelli et al. 2013; Jelić et al. 2014, 2015; Lenc et al. 2016; van Eck et al. 2017).

Two other pieces of this puzzle are pulsar RMs and the large samples of extragalactic RMs that will be available soon, e.g., from the POSSUM survey with the Australian Square Kilometre Array Pathfinder (Gaensler 2009). In this paper we make an attempt to compare and contrast these three sets of data on Faraday rotation, starting with latitudes above $\sim 25^{\circ}$, 

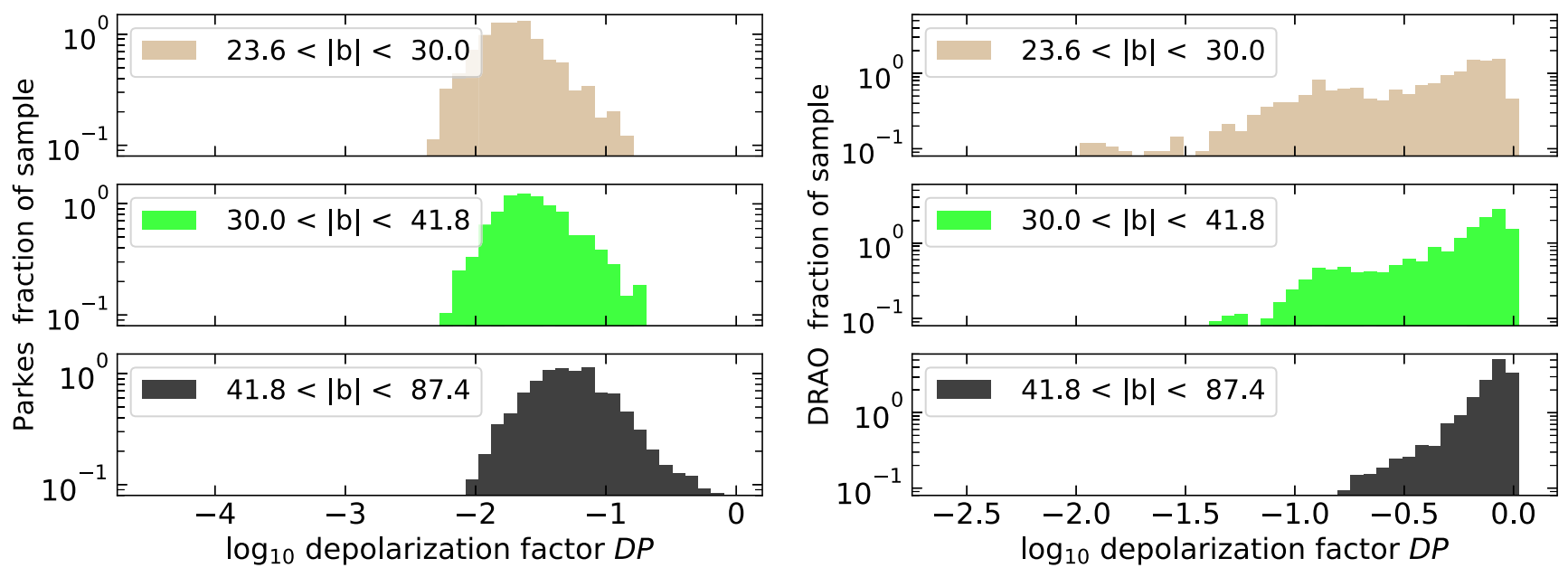

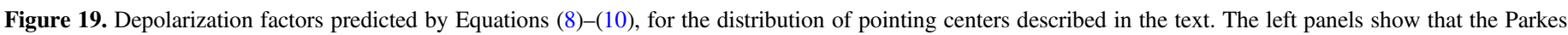

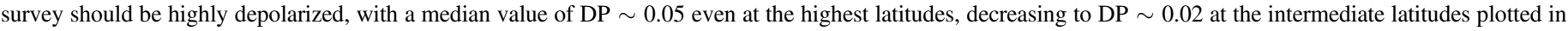

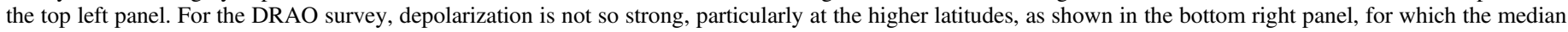
value of DP is $\sim 0.77$, decreasing to DP $\sim 0.29$ at the intermediate latitudes shown in the top right panel.

where the path length through the magneto-ionic medium is short. There are not quite enough pulsars to determine distances to specific features in the Faraday spectra, but there are fairly strong correlations between the pulsar and extragalactic foreground RMs and between the nearby pulsars and the first moments of the Faraday spectra from the DRAO survey.

The spectra from the Parkes survey have much better resolution in $\phi$, and they show compelling detail that will someday be traceable to structures in the nearby interstellar medium, most likely at distances of a few hundred parsecs or less. Some of these can be associated with known structures, including H II regions (Thomson et al. 2018b, 2018a; for other examples, see Gaensler et al. 2001; Madsen et al. 2006; Harvey-Smith et al. 2011) and neutral interstellar clouds (van Eck et al. 2017). But at the low frequencies of the Parkes survey, magnetized plasma that has a significant effect on the Faraday spectrum can be so diffuse as to be completely undetectable in $\mathrm{H} \alpha$ or any other spectral line tracer at any wavelength. Thus, as low-frequency polarization surveys like the Parkes survey improve, they will reveal more and more of the structure of the local interstellar diffuse ionized medium.

Based on the pulsar correlation with the first moments of the DRAO survey in Figure 14 and Table 3, we find the best correlation for a sample with maximum pulsar distance $700 \mathrm{pc}$. The correlation for a sample with maximum distance of $1 \mathrm{kpc}$ is significantly worse. The absence of correlation between the first moment of the Parkes survey data and the RMs of pulsars in any distance sample suggests that the polarized emission seen in that survey is mostly within about $300 \mathrm{pc}$, but the number of pulsars closer than this (five in the DRAO survey area and seven in the Parkes survey area; Table 3) is too small to search for correlations effectively. The comparison of the Parkes second moment with the dispersion of the pulsar RMs in Figure 15 also suggests a distance less than 500 pc.

A more sophisticated approach to determine, or at least to set limits on, the polarization horizon in the Parkes survey is to simulate the random magnetic field based on its turbulent spectrum, apply an electron density model, and compute typical values of $\phi$ (Hill 2018). That is beyond the scope of this paper, but several recent studies have reported impressive results that could be applied to the GMIMS survey, including
Herron et al. (2016, 2018a, 2018b) and Beck et al. (2016); see also the statistical approach used by Iacobelli et al. (2014).

The behavior of the different moments of the Faraday spectra versus path length $(\operatorname{cosec}|b|)$ is consistent with a paradigm where the long-wavelength polarization (Parkes data) is coming from a relatively small volume around the Sun, considerably smaller than the scale height of the magneto-ionic medium. In this region there is evidence for a vertical component of the $B$ field at high latitudes, with the field pointing toward the Sun from both the north and south Galactic poles (Figure 8, right panel). A similar trend is not seen in the DRAO survey first moments, so this is apparently a local phenomenon. On the longer lines of sight sampled by the DRAO survey the first moments indicate a $\boldsymbol{B}$-field component in the $-\hat{z}$-direction, i.e., from the northern to the southern Galactic hemispheres (Figure 8, left panel). This is a small effect; at high and intermediate latitudes random variations of the field lead to a dispersion in the measured first moments that is generally on the same order as the systematic effect (Figure 16). But the trend of the first moments with latitude is confirmed by similarly placed samples of the RM-foreground map from Oppermann et al. (2015) (Figure 11). The second moments of the DRAO survey increase as the square root of the number of $B$-field structures, as in a random walk process, hence the correlation of $m_{2}$ with $\operatorname{cosec}|b|$ (left panel of Figure 9), although the less well sampled southern hemisphere points show a weak opposite trend.

The ultimate significance of the GMIMS survey data will depend on how much it influences the development of comprehensive models of the Galactic magnetic field and the related physics of cosmic-ray propagation, such as GALPROP (Strong et al. 2010; Grenier et al. 2015). An approach with a robust statistical basis is the IMAGINE Consortium Bayesian platform (Boulanger et al. 2018), which has the goal to unify observations of many different kinds. Simulations of the magneto-ionic medium to predict and study the results of RM surveys are showing which analysis techniques are most robust and revealing (Haverkorn et al. 2008; Beck et al. 2016; Herron et al. 2018a; Reissl et al. 2018). As rapid progress is made in the field of Faraday spectroscopy, we can hope for improved 
models of the nearby magnetic field and its interaction with structures in the ionized interstellar medium.

The Parkes Radio Telescope is part of the Australia Telescope National Facility, which is funded by the Commonwealth of Australia for operation as a national facility managed by CSIRO. The DRAO $26 \mathrm{~m}$ telescope is operated as a national facility by the National Research Council Canada. The Dunlap Institute is funded through an endowment established by the David Dunlap family and the University of Toronto. We are grateful to JinLin Han for providing his complete table of 1001 pulsars with RM and distance measurements. We are grateful to Rainer Beck for a critical reading of the manuscript and helpful suggestions. J.D. is grateful for the hospitality of the Boston University Center for Astrophysical Research, where some of this work was done. A.T. acknowledges the support of an Australian Government Research Training Program (RTP) Scholarship. This research made use of Astropy, ${ }^{14}$ a community-developed core Python package for Astronomy (Astropy Collaboration et al. 2018).

\section{Appendix A Advantages of Spectral Moments versus Two Alternatives}

The spectral moment analysis in this paper is one approach to simplify and convey the information contained in the Faraday cube in the form of a small number of 2D images or maps. In ordinary spectroscopic imaging, e.g., with an aperture synthesis telescope observing a spectral line in emission, the moments are useful to characterize the kinematics of the source. The first moment may be used to trace the radial velocity field, the second moment the turbulence, and the zero moment often gives the column density of the atoms or molecules emitting the line. Rotation curves of galaxies are usually fitted to the first-moment map. An alternative approach that is sometimes simpler is to fit a Gaussian line profile to the spectrum at each pixel and use the resulting maps of the peak, center, and width $\left(T_{o}, \phi_{o}\right.$, and $\sigma_{\phi}$ in the notation of Equation (4)) to characterize the velocity field and to study the variation in line width from point to point. An even simpler approach is simply to find the highest point on the spectrum, $T_{\text {peak }}$, and the corresponding velocity, or in our case Faraday depth, $\phi_{\text {peak }}$.

Most previous surveys of Galactic synchrotron polarization at frequencies above $250 \mathrm{MHz}$ have suffered from observing too narrow a range of wavelengths, $\Delta \lambda^{2}$, so that the width of the RMSF, $\delta \phi$, is very broad (Table 1 ). The result is that the Faraday spectrum is convolved with a very broad Gaussian that smooths away the detailed structure of $T(\phi)$. This can be seen in the black traces in Figures 2-4 corresponding to the DRAO survey data; since $\delta \phi=140 \mathrm{rad} \mathrm{m}^{-2}$ for the DRAO survey, the spectrum is effectively convolved with a broad smoothing function. There is structure in the spectra in some directions that is broader than this width, but mostly the observed spectra in the DRAO survey could be approximately fitted by Gaussians without losing much information. Simply measuring the peak of the spectrum and its RM gives a quick characterization of the strength of the polarization and a single value for the RM. This is the way that polarization surveys were done in the last century, where a single value of the polarized brightness temperature and a single RM were

\footnotetext{
14 http://www.astropy.org
}

calculated over a narrow bandwidth at a given center frequency.

For polarization surveys like GMIMS, which are attempting to measure the Faraday spectrum with $\delta \phi$ small enough to show detailed line shapes like those seen in the red profiles in Figures 2-4 from the Parkes survey, a more subtle approach is needed to characterize the distribution of the emission over $\phi$ at each position. The Faraday moments are a good tool for this if there is more than one feature present in the spectrum. Figure 20 shows three maps of the Parkes survey data, illustrating the effect of taking the first moment, fitting Gaussians and making a map from the fitted center $\left(\phi_{o}\right)$, and simply finding the peak and plotting the resulting value of $\phi_{\text {peak }}$. The left panel is an expanded version of the bottom panel of Figure 6, showing the first moment of the Parkes survey on an area of the inner Galaxy with $-40^{\circ}<(\ell, b)<+40^{\circ}$. The middle panel shows the center value, $\phi_{o}$, of a Gaussian fitted to the channels of the Faraday spectrum above the threshold used in the moment calculation, and the right panel shows the value of $\phi_{\text {peak }}$ of the highest channel of the spectrum.

The two positions shown in the spectra in Figure 2 are indicated by black and white markers in each panel of Figure 20, at (longitude, latitude $)=(10.72,+35.47)$ and $(30.51,-33.08)$. The values at the centers of the markers are $(-1.2,-3.1,-3.5) \mathrm{rad} \mathrm{m}^{-2}$ for the former position and $(-1.0$, $-1.9,-4.5)$ for the latter for the left, middle, and right panels. Over the entire area shown in Figure 20, the mean and standard deviation of the difference between the mean $\phi$ calculated using the moment formula and the Gaussian fitted $\phi_{o}$ are 0.09 and $1.80 \mathrm{rad} \mathrm{m}^{-2}$. The mean difference between the first moment and the peak $\phi$ over this area is $0.13 \mathrm{rad} \mathrm{m}^{-2}$ with standard deviation $2.6 \mathrm{rad} \mathrm{m}^{-2}$. These differences are small, but the first-moment calculation takes account of the structure of the Faraday spectrum more carefully than taking $\phi_{\text {peak. This }}$ makes a difference as long as the spectrum has not been heavily smoothed by a broad RMSF. The GMIMS survey is designed to minimize $\delta \phi$ by using wide-band receivers to cover a large fractional range of $\lambda$. The moment calculations make the most of this narrow Faraday spectral resolution. As van Eck et al. (2017) show, Galactic Faraday spectra measured with lowfrequency telescopes commonly exhibit two or more distinct components that can be identified with separate structures on the line of sight. Whenever the Faraday spectrum shows multiple features, the moment calculation gives a much better estimate of the center $\phi$ and $\phi$-width than a single Gaussian fit or simply the peak value.

Figure 21 shows a similar comparison of the zero-moment maps for the region shown in Figure 20. Here the fitted Gaussian parameters are combined to give the Gaussian integral $G 0=\sqrt{2 \pi} T_{o} \sigma_{\phi}$. The peak values are shown in the right panel scaled to match the zero moments as $10.4 T_{\text {peak }}=4.9 \cdot \sqrt{2 \pi} T_{\text {peak }}$, where 4.9 is the mean value of the half-width, $\sigma_{\phi}$, of the Gaussian fits in this area. There is a very good match between the integrals of the Gaussian fits (middle panel) and the zero-moment values (left panel); the highest point values (right panel) match pretty well with the other two. For the two pulsar positions in Figure 2 the numbers are $(2.8,2.3,3.7) \mathrm{K} \mathrm{rad} \mathrm{m}^{-2}$ for the northern point and (3.5, 3.6, 3.8) $\mathrm{K} \mathrm{rad} \mathrm{m}^{-2}$ for the southern point. For the entire area, the difference between the calculated zero-moment value (left panel, Figure 21) and the peak value (right panel) has standard deviation $2.7 \mathrm{~K} \mathrm{rad} \mathrm{m}^{-2}$. The Gaussian integral (middle panel) 


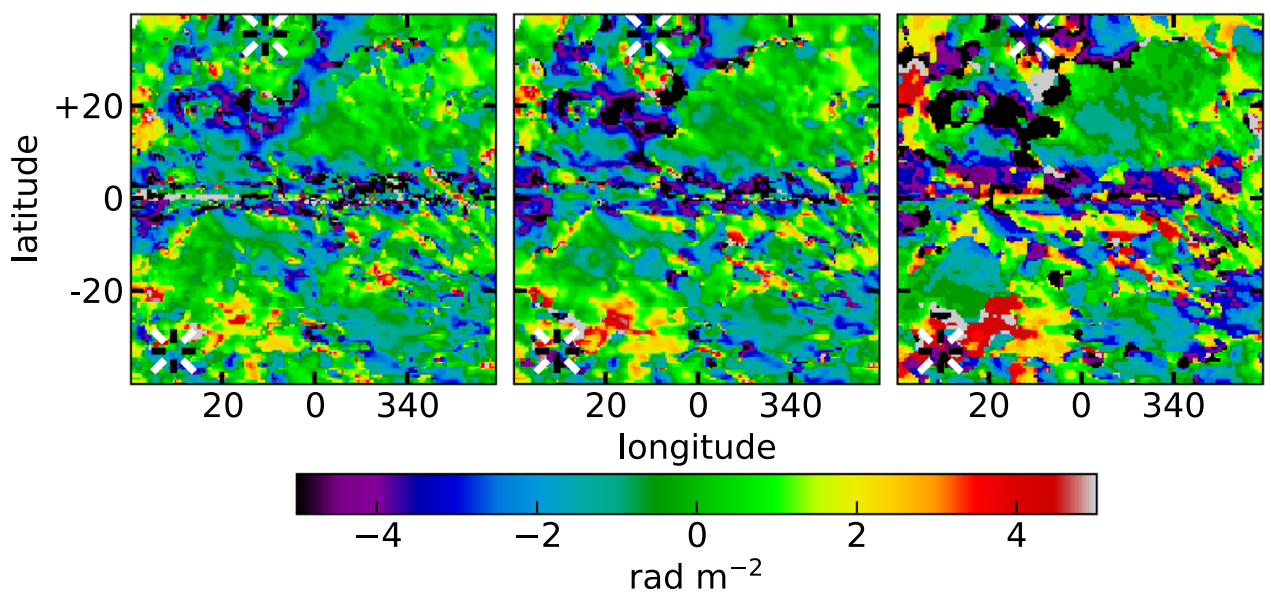

Figure 20. Comparison of three different methods of calculating the central RM of a Faraday spectrum of polarized emission. Left: first-moment map of a section of the Parkes survey (taken from Figure 6, bottom panel). Middle: same area, with the center value, $\phi_{o}$, of a Gaussian fit to each pixel. Right: value of $\phi_{\text {peak }}$ for the center of the channel with the peak or highest value of $T(\phi)$. Markers in the lower left corners and near the top, left of center, show the positions of the spectra illustrated in Figure 2.
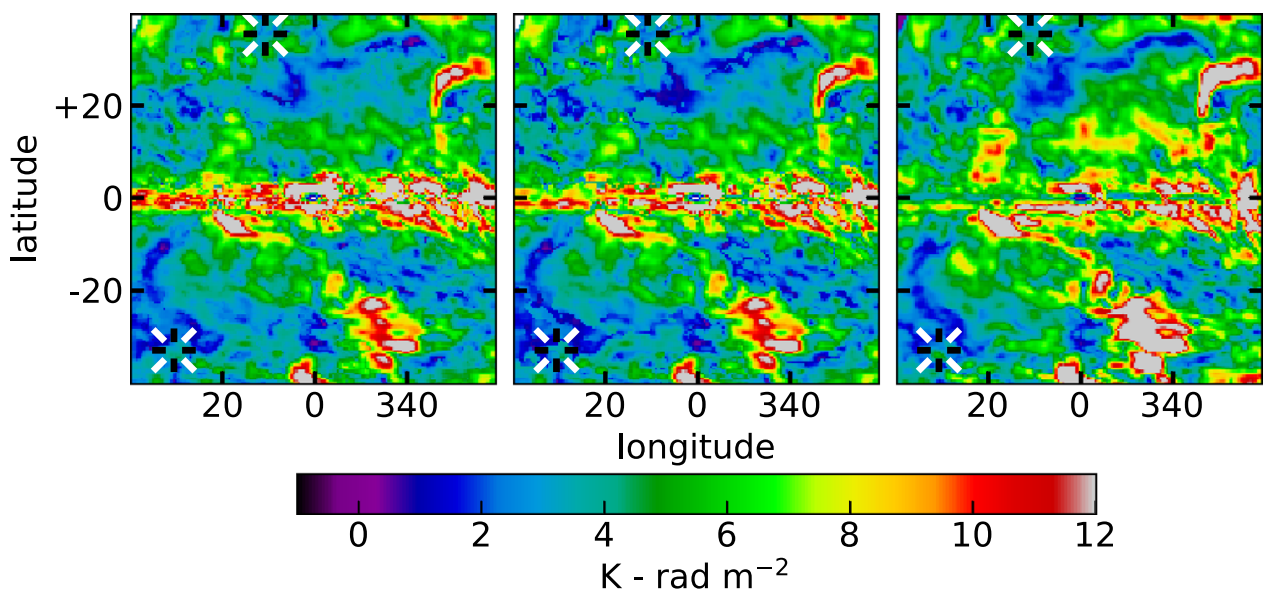

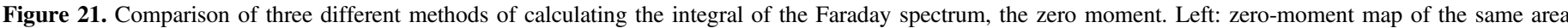

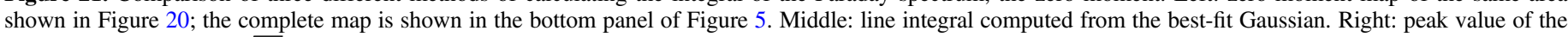
spectrum, multiplied by $\sqrt{2 \pi} \overline{\sigma_{o}}$, where $\overline{\sigma_{o}}=4.9$ is the mean value over this area.

matches the zero moment much better, their difference has standard deviation $0.76 \mathrm{~K} \mathrm{rad} \mathrm{m}^{-2}$. Since the peak value does not take into account the width of the Faraday spectral feature (s), it is not surprising that it gives a rougher estimate of the total linearly polarized brightness temperature. The peak value formally equals the brightness temperature of the polarized emission at just the single RM $(\phi)$ corresponding to $\phi_{\text {peak }}$. Faraday spectroscopy allows the separation of many different contributions to the observed spectrum of linear polarized brightness, each with a different RM. The spectral moment calculation is designed to capture the richness of the resulting Faraday cube.

\section{Appendix B \\ The Effect of Missing Short Wavelengths on the Faraday Spectral Moments}

The shortest wavelengths in a polarization survey limit the sensitivity to broad features in the Faraday spectrum, as discussed in Section 1.3. The values of $\lambda_{1}^{2}$ for the Parkes and DRAO surveys (Table 1) lead to $\phi_{\text {max-scale values of } 8.0}$ and $110 \mathrm{rad} \mathrm{m}^{-2}$. This is much less than the maximum RM detectable, which is set by the width of the spectrometer channels, $\Delta \lambda^{2}$. The two surveys are sensitive to features in the Faraday spectrum up to very high values $\left(>10^{3} \mathrm{rad} \mathrm{m}^{-2}\right)$, much more than needed for a survey of high and intermediate latitudes in the Milky Way. Thus, there is no bias against detecting features at high values of $\phi$ in our spectra. But there is a strong bias in the Parkes survey against detecting broad features centered at any value of $\phi$. This effect has been analyzed in several papers, starting with Brentjens \& de Bruyn (2005); other illustrations are given by Frick et al. (2011) and Beck et al. (2012). Here we consider the effect of the missing short-wavelength data on the moments calculated as described in Section 2.3.

The simplest line profile function to consider is a Gaussian. If a Faraday spectral feature has a Gaussian shape in $\phi$ space, then it will have a Gaussian shape in $\lambda^{2}$ space as well. Figure 22 shows the effect of the missing short wavelengths on progressively broader Gaussian features. On the left side are Faraday spectra, on the right the corresponding spectra in the $\lambda^{2}$ space. The calculations are made with $10^{3}$ equally spaced channels, but the figure expands the ranges of significance on 

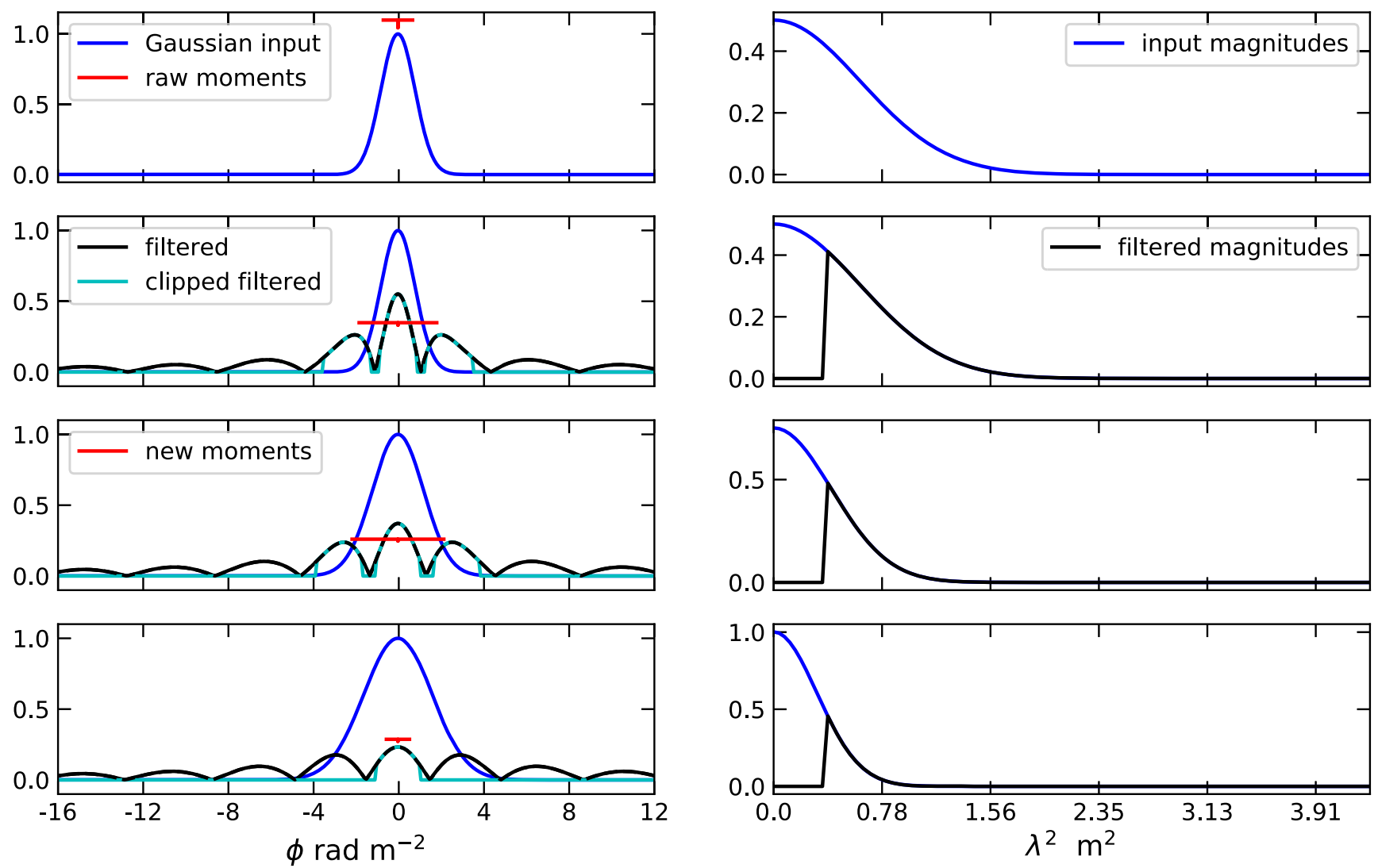

Figure 22. Effect of the missing short wavelengths on a Gaussian line profile in Faraday space, and the resulting moments calculated after clipping the filtered profile. The blue curves in the panels on the left side are Gaussian-shaped Faraday line profiles, $F(\phi)$, and the corresponding blue Gaussians in the right panels are their transforms to $\lambda^{2}$ space by Equation (2). In the second, third, and fourth rows are shown in black the profiles after filtering out the short wavelengths, $\lambda^{2}<0.391 \mathrm{~m}{ }^{2}$, corresponding to the Parkes survey maximum frequency of $480 \mathrm{MHz}$. Thresholding as described in Section 2.4 changes the black curves in the left panels to the green curves, and the moments calculated after thresholding are shown by the red bars, as in Figures 2-4.

both sides for clarity. The $y$ scales are in arbitrary units, with zero points indicated.

In Figure 22 the top row shows a complete Gaussian on both sides, the ideal case with no missing short wavelengths. The second through fourth rows show the effect of a gap in the measured values of $P\left(\lambda^{2}\right)$ for progressively broader features in the Faraday spectrum, $F(\phi)$ (Equation (2)). The left panels for each row show the effect of this filtering on the Faraday profile function, before and after the clipping applied in the moments calculation, and the red bars above the lines show the resulting moments. The zeroth moment is translated into an equivalent line peak by the Gaussian formula $T_{0}=M_{0} /\left(\sqrt{2 \pi} \sigma_{\phi}\right)$, as in Figures 2-4. As the feature width grows from $\sigma_{\phi}=8$ to 12 and then to $16 \mathrm{rad} \mathrm{m}^{-2}$, on the second, third, and fourth rows of Figure 22, the width of the feature in $\lambda^{2}$ conjugate space narrows. The black curves in the right panels show the effect of the missing short wavelengths on the line profile in $\lambda^{2}$, and the black and green curves in the corresponding panels on the left side show the effect of this filtering on the line profile in Faraday space $(\phi)$. The green curve, which partially covers the black curve, shows the result after the clipping at $15 \%$ of the peak value, applied in the calculation of the moments. The red bars above the profiles show the values of the first and second moments that would then be calculated from the filtered, clipped line profile in $\phi$.

An alternate profile shape that has been considered by several authors is a boxcar or top-hat function, shown in
Figure 23. Here the effect of the missing short wavelengths is dramatic, because the discontinuous edges of the boxcar become two spikes after filtering. The second moment, $m 2$, is not very sensitive to the filtering in this case, because the spacing between the two spikes or horns on the filtered profile does not change much. The zero moment for the functions shown in Figure 23 is not much affected either, because this is the integral of the magnitude of $T_{\mathrm{pol}}(\phi)$.

The effects of the filtering and clipping on the computed values of moment zero and moment 2 are shown for a wider range of widths of boxcar functions in Figure 24. The curves show the ratios of the computed values of $M_{0}$ and $m 2$ to their values for a simple boxcar of the same width, as a function of width. The clipping alone (blue and green lines) makes almost no difference at all, since a clipped boxcar is the same as a boxcar, but the filtering increases and then decreases $M_{0}$ as the line width increases. The filtering greatly increases the second moment; even for the broadest lines the effect is a factor of two, and for narrower lines it is as high as 3.5.

Similar to Figure 24 is Figure 25, but for Gaussian line profiles similar to those in Figure 22. Here the clipping has a weak effect in reducing both $M_{0}$ and $m 2$, but the filtering effect is much more severe. On the right (widest input Gaussians) the filtering has attenuated the line below the threshold at all values of $\phi$, so that both moments are zero. For narrower input Gaussians, the filtering increases $m 2$, because the main line is surrounded by sidebands or spurious secondary features on either side. In a more realistic case of an asymmetric line 

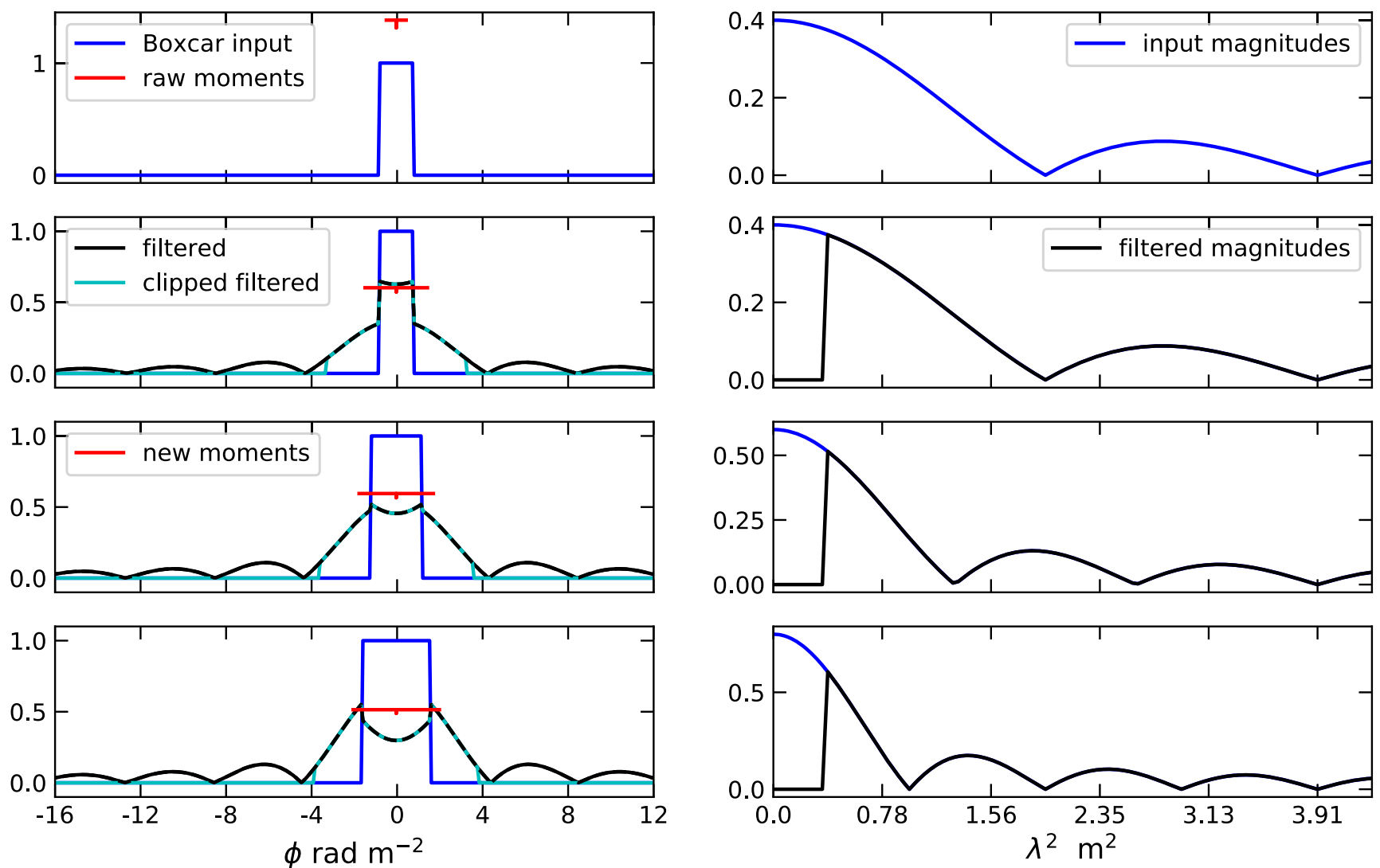

Figure 23. Effect of the missing short wavelengths on a boxcar line profile in Faraday space, and the resulting moments calculated after clipping the filtered profile. The colors and layout are similar to those in Figure 22. Because of its discontinuous edges, the boxcar function turns into a "two-horned" profile after heavy filtering. This is probably unrealistic; the Faraday profile of a slab of mixed synchrotron emission and magnetized plasma would have smooth, continuous edges as a result of irregularities in the density and $\boldsymbol{B}$ fields. This will lead to profiles more like the Gaussians in Figure 22.

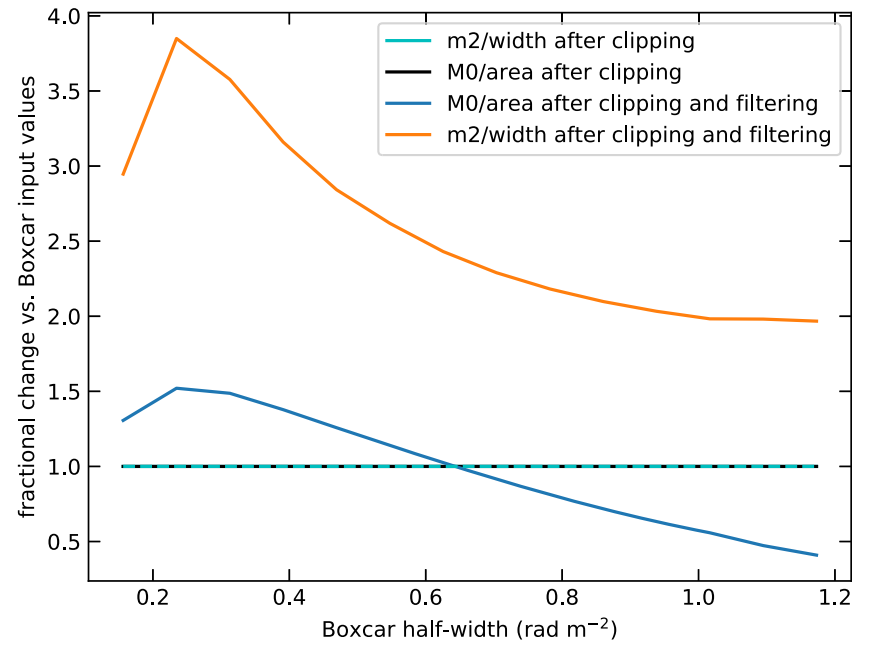

Figure 24. Effect of the filtering caused by the missing short wavelengths on the measured moments of synthetic spectra like those in Figure 23. The width of the boxcar increases from left to right on the $x$-axis, and the ratio of the values of $M_{0}$ and $m 2$ measured for the filtered, clipped spectrum to their corresponding values for the original boxcar is shown on the $y$-axis. As discussed in the text, the result of the filtering due to missing short wavelengths for narrow input lines is to more than triple the apparent width of the line. As the input line width increases, both moments decrease owing to the filtering. The curves show the ratio of the moments $\left(M_{0}\right.$ and $\left.m 2\right)$ measured on the clipped and filtered Faraday spectra to the input values of the area under the boxcar, i.e., the area or true value of the moment $M_{0}$, and the half-width of the boxcar, i.e., "width," which is the true value of $m 2$.

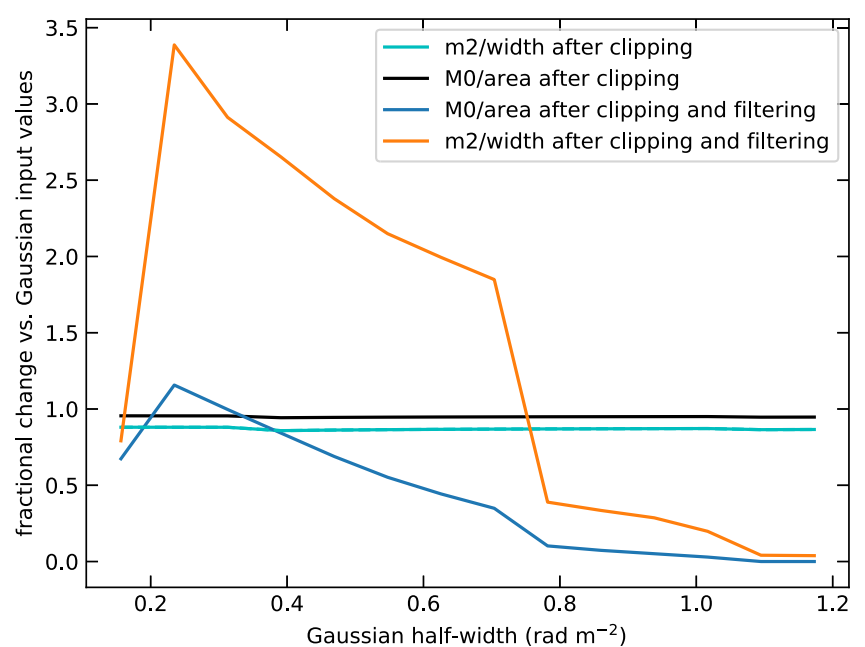

Figure 25. Effect of the filtering caused by the missing short wavelengths on the measured moments of synthetic Gaussian spectra like those in Figure 22. The width of the Gaussian increases from left to right on the $x$-axis, and the ratio of the values of $M_{0}$ and $m 2$ measured for the filtered, clipped spectrum to their corresponding values for the original Gaussian is shown on the $y$-axis. As discussed in the text, the result of the filtering due to missing short wavelengths for narrow input lines is to more than triple the apparent width of the line. As the input line width increases, both moments decrease owing to the filtering. The curves show the ratio of the measured moments $\left(M_{0}\right.$ and $\left.m 2\right)$ to the corresponding values for the input function, i.e., the area under the Gaussian, $M_{0}$, and the width of the Gaussian, $\sigma=m_{2}$. 
profile, it is likely that only one of the sidebands would be above the threshold, leading to a smaller increase in the measured value of $m 2$. It is common in the Parkes spectra to see features with two peaks, which may be the result of the missing short-wavelength data.

The ultimate goal of the GMIMS collaboration is to combine surveys with different telescopes that will cover the full wavelength range from $\lambda \sim 1 \mathrm{~m}$ to $\lambda \sim 10 \mathrm{~cm}$, so as to be sensitive to the full range of feature widths in the Faraday spectrum. The Parkes and DRAO surveys are the first big steps toward that important objective. When it is achieved, it will provide an excellent view of the Galactic magneto-ionic medium that cannot be traced in any other way.

\section{ORCID iDs}

John M. Dickey (iD https://orcid.org/0000-0002-6300-7459 Alec J. M. Thomson (D) https://orcid.org/0000-00019472-041X

E. Carretti (iD https://orcid.org/0000-0002-3973-8403

A. Fletcher (i) https://orcid.org/0000-0003-1741-1714

B. M. Gaensler (D) https://orcid.org/0000-0002-3382-9558

M. Haverkorn (iD https://orcid.org/0000-0002-5288-312X

A. S. Hill (i) https://orcid.org/0000-0001-7301-5666

N. M. McClure-Griffiths (iD) https://orcid.org/0000-00032730-957X

\section{References}

Alfvén, H., \& Herlofsen, N. 1950, PhRv, 73, 616

Alves, M. I. R., Boulanger, F., Ferrière, K., \& Montier, L. 2018, A\&A, 611, L5 Astropy Collaboration, Price-Whelan, A. M., Sipocz, B. M., et al. 2018, AJ, 156,123

Beck, M. C., Beck, A. M., Beck, R., et al. 2016, JCAP, 5, 056

Beck, R., Frick, P., Stapanov, R., \& Sokoloff, D. 2012, A\&A, 543, 113

Boulanger, F., Ensslin, R., Fletcher, A., et al. 2018, arXiv:1805.02496

Brentjens, M. A., \& de Bruyn, A. G. 2005, A\&A, 441, 1217

Brouw, W. N., Muller, C. A., \& Tinbergen, J. 1962, BAN, 518, 213

Burn, B. J. 1966, MNRAS, 133, 67

Clark, S. E. 2018, ApJL, 857, 10

Clemens, D. P., Pavel, M. D., \& Cashman, L. R. 2012, ApJS, 200, 21

Crutcher, R. M., Wandelt, B., Heiles, C., Falgarone, E., \& Troland, T. H. 2010, ApJ, 725, 466

de Bruyn, A. G., \& Brentjens, M. A. 2005, A\&A, 441, 931

Farnes, J. S., Heald, G., Junklewitz, H., et al. 2018, MNRAS, 474, 3280

Ferrière, K. 2015, J. Ph. Conf. Ser., 577, 012008

Ferrière, K. M. 2001, RvMP, 73, 1031

Frick, P., Sokoloff, D., Stepanov, R., \& Beck, R. 2011, MNRAS, 414, 2540

Frisch, P. C., Andersson, B., Berdyugin, A., et al. 2012, ApJ, 760, 106

Gaensler, B. M. 2009, in IAUS 259, Cosmic Magnetic Fields: From Planets, to Stars and Galaxies, ed. K. G. Strassmeier, A. G. Kosovichev, \& J. E. Beckman (Cambridge: Cambridge Univ. Press), 645

Gaensler, B. M., Dickey, J. M., McClure-Griffiths, N. M., et al. 2001, ApJ, 549, 959

Gaensler, B. M., Madsen, G. J., Chatterjee, S., \& Mao, S. A. 2008, PASA, 25,184

Ginzburg, V. L., \& Syrovatskii, S. I. 1965, ARA\&A, 3, 297

Grenier, I. A., Black, J. H., \& Strong, A. W. 2015, ARA\&A, 53, 199

Hall, J. S. 1949, Sci, 109, 166

Han, J. L. 2017, ARA\&A, 55, 111

Han, J. L., Manchester, R. N., Lyne, A. G., Qiao, G. J., \& van Straten, W. 2006, ApJ, 642, 868

Han, J. L., Manchester, R. N., van Straten, W., \& Demorest, P. 2018a, ApJS, 234,11

Han, J. L., Manchester, R. N., van Straten, W., \& Demorest, P. 2018b, yCat, 22340011
Harvey-Smith, L., Madsen, G. J., \& Gaensler, B. M. 2011, ApJ, 736, 83 Harwit, M. 1973, Astrophysical Concepts (New York: Wiley), 6

Haverkorn, M., Brown, J. C., Gaensler, B. M., \& McClure-Griffiths, N. M. 2008, ApJ, 680, 362

Heald, G. 2009, in IAU Symp. 259, Cosmic Magnetic Fields: From Planets, to Stars and Galaxies, ed. K. G. Strassmeier, A. G. Kosovichev, \& J. E. Beckman (Cambridge: Cambridge Univ. Press), 591

Heiles, C. 2000, AJ, 119, 923

Herron, C. A., Burkhart, B., Gaensler, B. M., et al. 2018a, ApJ, 855, 29

Herron, C. A., Burkhart, B., Lazarian, A., Gaensler, B. M., \& McClure-Griffiths, N. M. 2016, ApJ, 822, 13

Herron, C. A., Gaensler, B. M., Lewis, G. F., \& McClure-Griffiths, N. M. 2018b, ApJ, 853, 9

Hill, A. S. 2018, Galaxies, 6, 129

Hill, A. S., Landecker, T. L., Carretti, E., et al. 2017, MNRAS, 467, 4631

Hiltner, W. A. 1949, ApJ, 109, 471

Houde, M., Rao, R., Vaillancourt, J. E., \& Hildebrand, R. H. 2011, ApJ, 733, 109

Iacobelli, M., Burkhart, B., Haverkorn, M., et al. 2014, A\&A, 566, A5

Iacobelli, M., Haverkorn, M., \& Katgert, P. 2013, A\&A, 549, A56

Jelić, V., de Bruyn, A. G., Mevius, M., et al. 2014, A\&A, 568, 101

Jelić, V., de Bruyn, A. G., Pandey, V. N., et al. 2015, A\&A, 583, 137

Jokipii, J. R., \& Lerche, I. 1969, ApJ, 157, 1137

Jones, M. E., Taylor, A. C., Aich, M., et al. 2018, MNRAS, 480, 3224

Jones, T. J. 2003, AJ, 125, 3208

Lenc, E., Gaensler, M. M., Sun, X. H., et al. 2016, ApJ, 830, 38L

Madsen, G. J., Reynolds, R. J., \& Haffner, L. M. 2006, ApJ, 652, 401

Manchester, R. N., Hobbs, G. B., Teoh, A., \& Hobbs, M. 2005, AJ, 129 1993

Mao, S. A. 2018, arXiv: 1810.03619

Mao, S. A., Gaensler, B. M., Haverkorn, M., et al. 2010, ApJ, 714, 1170

Mao, S. A., McClure-Griffiths, N. M., Gaensler, B. M., et al. 2012, ApJ, 755,21

Mao, S. A., McClure-Griffiths, N. M., Gaensler, B. M., et al. 2015a, HiA, 16,403

Mao, S. A., Zweibel, E., Fletcher, A., Ott, J., \& Tabatabaei, F. 2015b, ApJ, 800,92

Mathewson, D. S., \& Ford, V. I. 1970, MmRAS, 74, 139

Miville-Deschênes, M.-A., Ysard, N., Lavabre, A., et al. 2008, A\&A, 490, 1093

Oppermann, N., Junklewitz, H., Greiner, M., et al. 2015, A\&A, 575, A118

Oppermann, N., Junklewitz, H., Robbers, G., et al. 2012, A\&A, 542, A93

Ordog, A., Brown, J. C., Kothes, R., \& Landecker, T. L. 2017, A\&A, 603, 15

O'Sullivan, S. P., Purcell, C. R., Anderson, C. S., et al. 2017, MNRAS, 469, 4034

Page, L., Hinshaw, G., Komatsu, E., et al. 2007, ApJS, 170, 335

Planck Collaboration, Aghanim, N., Akrami, Y., et al. 2018, A\&A, in press (arXiv:1807.06212)

Reissl, S., Stutz, A. M., Brauer, R., et al. 2018, MNRAS, 481, 2507

Schnitzeler, D. H. F. M. 2010, MNRAS, 409, L99

Schnitzeler, D. H. F. M. 2018, MNRAS, 474, 300

Schnitzeler, D. H. F. M., \& Lee, K. J. 2015, MNRAS, 447, L26

Sokoloff, D. D., Bykov, A. A., Shukurov, A., et al. 1998, MNRAS, 299 189

Stil, J., Taylor, A. R., \& Sunstrum, C. 2011, ApJ, 726, 4

Strong, A. W., Porter, T. A., Digel, S. W., et al. 2010, ApJL, 722, 58

Su, H., Macquart, J., Hurley-Walker, N., et al. 2018, MNRAS, 479, 4041

Sun, X. H., Han, J. L., Reich, W., et al. 2007, A\&A, 463, 993

Sun, X. H., Landecker, T. L., Gaensler, B. M., et al. 2015, ApJ, 811, 40

Taylor, A. R., Stil, J. M., \& Sunstrum, C. 2009, ApJ, 702, 1230

Thomson, A. J. M., Landecker, T. L., Dickey, J. M., et al. 2018a, in press

Thomson, A. J. M., McClure-Griffiths, N. M., Federrath, C., et al. 2018b, MNRAS, 479, 5620

Tribble, P. C. 1991, MNRAS, 150, 726

Uyaniker, B., Landecker, T. L., Gray, A. D., \& Kothes, R. 2003, ApJ, 585, 785

van Eck, C., Haverkorn, M., Alves, M. I. R., et al. 2017, A\&A, 597, 98

Wolleben, M., Fletcher, A., Landecker, T. L., et al. 2010, ApJ, 724, 48

Wolleben, M., Landecker, T. L., Carretti, E., et al. 2018, ApJ, submitted

Wolleben, M., Landecker, T. L., Caretti, E., et al. 2009, IAUS, 259, 539

Yao, J. M., Manchester, R. N., \& Wang, N. 2017, ApJ, 835, 29

Zamora-Avilès, M., Vásquez-Semadeni, E., Körtgen, B., Banerjee, R., \& Hartmann, L. 2018, MNRAS, 474, 4824 